TRANSACTIONS OF THE

AMERICAN MATHEMATICAL SOCIETY

Volume 364, Number 7, July 2012, Pages 3395-3425

S 0002-9947(2012)05383-9

Article electronically published on February 20, 2012

\title{
LOCAL REGULARITY AND DECAY ESTIMATES OF SOLITARY WAVES FOR THE ROTATION-MODIFIED KADOMTSEV-PETVIASHVILI EQUATION
}

\author{
ROBIN MING CHEN, YUE LIU, AND PINGZHENG ZHANG
}

\begin{abstract}
This paper is mainly concerned with the local low regularity of solutions and decay estimates of solitary waves to the rotation-modified Kadomtsev-Petviashvili (rmKP) equation. It is shown that with negative dispersion, the rmKP equation is locally well-posed for data in $H^{s_{1}, s_{2}}\left(\mathbb{R}^{2}\right)$ for $s_{1}>-\frac{3}{10}$ and $s_{2} \geqslant 0$, and hence globally well-posed in the space $L^{2}$. Moreover, an improved result on the decay property of the solitary waves is established, which shows that all solitary waves of the rmKP equation decay exponentially at infinity.
\end{abstract}

\section{INTRODUCTION}

The rotation-modified Kadomtsev-Petviashvili (rmKP) equation

$$
\left(u_{t}-\beta u_{x x x}+\left(u^{2}\right)_{x}\right)_{x}+u_{y y}-\gamma u=0
$$

is a model [14, 15] to describe small-amplitude, long internal waves in a rotating fluid propagating in one dominant direction with slow transverse effects, where the effects of rotation balance with weakly nonlinear and dispersive effects. Here, $u(t, x, y)$ can be considered as the free surface, $t \in \mathbb{R}^{+}$is a timelike variable, $x \in \mathbb{R}$ is a spatial variable in the dominant direction of wave propagation, and $y \in \mathbb{R}$ is a spatial variable in a direction transverse to the $x$-direction. The parameter $\gamma$ measures the effects of rotation and is proportional to the Coriolis force. The high-dispersion parameter $\beta \neq 0$ determines the type of dispersion. In case $\beta<0$ (negative dispersion), the equation models gravity surface waves in a shallow water channel and internal waves in the ocean, while in case $\beta>0$ (positive dispersion) it models capillary waves on the surface of a liquid or oblique magneto-acoustic waves in plasma.

Setting $\gamma=0$, equation (1.1) becomes the well-known Kadomtsev-Petviashvili (KP) equation 21]

$$
\left(u_{t}-\beta u_{x x x}+\left(u^{2}\right)_{x}\right)_{x}+u_{y y}=0
$$

which arises in modeling the propagation of weakly nonlinear dispersive long waves on the surface of fluid, when the wave motion is essentially one-directional with weak transverse effects along the $y$-axis. Equation (1.2) with $\beta>0$ is known as KP-I, while its version with $\beta<0$ is called KP-II and is integrable by the inverse scattering method [1].

Received by the editors December 21, 2009 and, in revised form, April 19, 2010.

2010 Mathematics Subject Classification. Primary 35Q53, 76B03, 75B15, 76B25.

Key words and phrases. Kadomtsev-Petviashvili, rotation, Cauchy problem, well-posedness, solitary wave.

(C)2012 American Mathematical Society Reverts to public domain 28 years from publication 
In the first part of this paper we consider the Cauchy problem for equation (1.1). It has been shown in [10] by using the parabolic regularization and a compactness argument that equation (1.1) is locally well-posed for data in the space $X_{s}$ for $s>2$, where $X_{s}$ with $s \geqslant 0$ is defined as

$$
X_{s}=\left\{f \in H^{s}\left(\mathbb{R}^{2}\right):\left(\xi^{-1} \hat{f}\right)^{\vee} \in H^{s}\left(\mathbb{R}^{2}\right)\right\},
$$

with its norm

$$
\|f\|_{X_{s}}=\|f\|_{H^{s}}+\left\|\left(\xi^{-1} \hat{f}\right)^{\vee}\right\|_{H^{s}} .
$$

It is our purpose here to establish the local well-posedness result for equation (1.1) with data in an anisotropic Sobolev space of low order, so that one can hope to get the local and global low regularity of solutions. Studied here is the initial value problem of the form

$$
\left\{\begin{array}{l}
\left(u_{t}-\beta u_{x x x}+\left(u^{2}\right)_{x}\right)_{x}+u_{y y}-\gamma u=0, \quad(t, x, y) \in \mathbb{R}^{3}, \\
u(0, x, y)=\phi(x, y),
\end{array}\right.
$$

where the initial profile $\phi(x, y)$ belongs to an anisotropic Sobolev space $H^{s_{1}, s_{2}}\left(\mathbb{R}^{2}\right)$ defined by

$$
H^{s_{1}, s_{2}}\left(\mathbb{R}^{2}\right)=\left\{\phi \in \mathcal{S}^{\prime}\left(\mathbb{R}^{2}\right):\|\phi\|_{H^{s_{1}, s_{2}}}<\infty\right\}
$$

where

$$
\|\phi\|_{H^{s_{1}, s_{2}}}=\left\|\langle\xi\rangle^{s_{1}}\langle\eta\rangle^{s_{2}} \hat{u}(\xi, \eta)\right\|_{L^{2}}
$$

with $\langle\cdot\rangle=\left(1+|\cdot|^{2}\right)^{1 / 2}$.

The above spaces are a natural set for the initial data of the Cauchy problem for the Kadomtsev-Petviashvili equations because their homogeneous versions are invariant under the scale transformations preserving the KP equations. It has been understood that flows of the KP-I and KP-II equations, considered in the natural spaces, behave very differently. The KP-II equation can be solved by Picard iteration ([8]) while KP-I cannot, in any Sobolev class (24]). In [8], Bourgain proved the local (and therefore global due to the $L^{2}$ conservation law) well-posedness of the KP-II equation with $L^{2}$ initial data. Local and global well-posedness for the KP-II equation with data below $L^{2}$ were obtained in [20, 33, 34, 37. Their results were generalized in [17] to the sharp results in the critical space. All these results use the Fourier transform restriction method of Bourgain. On the other hand, it has been shown in 24] that the KP-I equation has a "bad behavior". In particular, the KP-I analogue of the crucial bilinear estimates established by Bourgain for the KP-II equation are proved to be false. In [11] and [38, a global existence result for small initial data was obtained via inverse scattering techniques. In 35 global existence of weak solutions in the energy space was established. The uniqueness of such solutions is unknown. The first result of global well-posedness of strong solutions to the KP-I equation was established in [25, 26. Using a method combining local well-posedness and delicate conservation laws, the authors managed to obtain the global well-posedness in the "second" energy space (see also [22]). Recently, Ionescu, Kenig and Tataru [18] obtained global well-posedness for the KP-I equation in the natural energy space by introducing some new methods which can be viewed as a combination of the Bourgain space method and energy estimates.

However, the rmKP does not preserve any scaling because of the rotation term. Hence it does not indicate any critical values for $\left(s_{1}, s_{2}\right)$ of the initial data in $H^{s_{1}, s_{2}}\left(\mathbb{R}^{2}\right)$ with which one may expect well-posedness. But when $\beta<0$ and $\gamma>0$, 
the solution operator $S(t)$ of the linear part of the equation has a good algebraic property similar to the one of the KP-II equation, which allows us to perform the Fourier transform restriction methods with some of the oscillatory integral estimates [4, 23]. More explicitly, the bilinear dispersive identity of the rmKP equation (1.1) is

$$
\begin{aligned}
p\left(\xi_{1}+\xi_{2}, \eta_{1}\right. & \left.+\eta_{2}\right)-p\left(\xi_{1}, \eta_{1}\right)-p\left(\xi_{2}, \eta_{2}\right) \\
& =-3 \beta \xi \xi_{1}\left(\xi-\xi_{1}\right)+\frac{\left(\xi_{1} \eta-\xi \eta_{1}\right)^{2}}{\xi \xi_{1}\left(\xi-\xi_{1}\right)}+\gamma \frac{\xi^{2}-\xi \xi_{1}+\xi_{1}^{2}}{\xi \xi_{1}\left(\xi-\xi_{1}\right)}
\end{aligned}
$$

where $p(\xi, \eta)$ is the associated dispersive function defined in (1.6). Clearly for $\gamma>0$, if $\beta>0$ (positive dispersion) this quantity could be zero, while if $\beta<0$ (negative dispersion)

$$
\left|p\left(\xi_{1}+\xi_{2}, \eta_{1}+\eta_{2}\right)-p\left(\xi_{1}, \eta_{1}\right)-p\left(\xi_{2}, \eta_{2}\right)\right| \geqslant C\left|\xi_{1} \xi_{2}\left(\xi_{1}+\xi_{2}\right)\right| .
$$

This, together with the Strichartz estimate, helps to control the derivative nonlinearity and to obtain well-posedness for rough data. But the payoff of the negative dispersion assumption is that we fail to establish a uniform time-decay estimate of the oscillatory integral associated to the linear problem, again due to the rotation term. This in turn prevents us from getting a uniform-in-time Strichartz estimate. This difficulty is resolved by breaking up the space frequency and performing the Strichartz estimate on each frequency part, as illustrated in Remark 1.1.

Motivated by 34, we define the following Bourgain spaces associated to equation (1.1).

Definition 1.1. For $b, b_{1}, b_{2}, s_{1}, s_{2} \in \mathbb{R}$, we define $X_{s_{1}, s_{2}}^{b, b_{1}, b_{2}}$ as a Bourgain-type space associated to the rmKP equation

$$
X_{s_{1}, s_{2}}^{b, b_{1}, b_{2}}=\left\{u \in \mathcal{S}^{\prime}\left(\mathbb{R}^{3}\right):\|u\|_{X_{s_{1}, s_{2}}^{b, b_{1}, b_{2}}}<\infty\right\},
$$

where

$$
\|u\|_{X_{s_{1}, s_{2}}^{b, b_{1}, b_{2}}}=\left\|\langle\tau-p(\xi, \eta)\rangle^{b}\langle\xi\rangle^{s_{1}}\langle\eta\rangle^{s_{2}}\left[1+\frac{\langle\tau-p(\xi, \eta)\rangle^{b_{1}}}{\langle\xi\rangle^{b_{2}}}\right] \hat{u}(\tau, \xi, \eta)\right\|_{L_{\tau \xi \eta}^{2}}
$$

with

$$
p(\xi, \eta)=-\beta \xi^{3}-\frac{\eta^{2}+\gamma}{\xi} .
$$

Let $I \subset \mathbb{R}$ be an interval. For $b>1 / 2$ we define the space $X_{s_{1}, s_{2}}^{b, b_{1}, b_{2}}(I)$ equipped with the norm

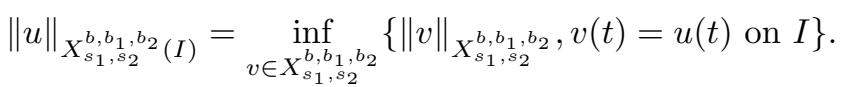

Equation (1.3) can be written as the integral equation

$$
u(t)=S(t) \phi-\int_{0}^{t} S\left(t-t^{\prime}\right)\left(u^{2}\left(t^{\prime}\right)\right)_{x} d t^{\prime},
$$

where $S(t)$ is the Fourier multiplier with symbol $e^{i t p(\xi, \eta)}$, with $p(\xi, \eta)$ given in (1.6).

In this paper we prove that with negative dispersion $\beta<0$, the $\operatorname{rmKP}$ equation (1.1) is locally well-posed for initial data in the anisotropic Sobolev space $H^{s_{1}, s_{2}}\left(\mathbb{R}^{2}\right)$ for $s_{1}>-3 / 10, s_{2} \geqslant 0$. The main tool for the proof of the local well-posedness of equation (1.1) is the following bilinear estimate. 
Theorem 1.2. Assume $\beta<0$ and $\gamma>0$. Let the real numbers $b, b^{\prime}, b_{1}, b_{2}, s_{1}, s_{2}$ be such that

$$
\begin{aligned}
& b_{1} \geqslant 0, \quad b_{2} \geqslant 0, \quad b>1 / 2, \quad s_{2} \geqslant 0, \quad s_{1}>b_{1}-b^{\prime}, \\
& s_{1}>1-3 b^{\prime}, \quad s_{1}>1+3 b_{1}-3 b^{\prime}-b_{2}, \quad b^{\prime}>b_{1}+1 / 4, \\
& b^{\prime}+5 b_{1}>\frac{3}{2}, \quad b^{\prime}+4 b_{1}+\frac{3}{2} b_{2}-4 b_{1} b_{2}>\frac{3}{2}, \quad s_{1}>b_{2}-1, \\
& b^{\prime}+b_{1}>1 / 2, \quad 2 b^{\prime}+b_{2}>1, \quad s_{1}>1-3 b_{1}-3 b^{\prime}+b_{2} .
\end{aligned}
$$

Then

$$
\left\|\partial_{x}(u v)\right\|_{X_{s_{1}, s_{2}}^{-b^{\prime}, b_{1}, b_{2}}} \leqslant C\|u\|_{X_{s_{1}, s_{2}}^{b, b_{1}, b_{2}}}\|v\|_{X_{s_{1}, s_{2}}^{b, b_{1}, b_{2}}} .
$$

Applying Theorem 1.2 with $b=\frac{1}{2}+, b^{\prime}=\frac{1}{2}-, b_{1}=\frac{1}{5}+\left(\right.$ depending on $\left.b^{\prime}\right)$, and $b_{2}=\frac{1}{3}$ we have

Theorem 1.3. Assume $\beta<0$ and $\gamma>0$. Let $s_{1}>-\frac{3}{10}$ and $s_{2} \geqslant 0$. Then

$$
\left\|\partial_{x}(u v)\right\|_{X_{s_{1}, s_{2}}^{-\frac{1}{2}+, \frac{1}{5}+, \frac{1}{3}}} \leqslant C\|u\|_{X_{s_{1}, s_{2}}^{\frac{1}{2}+, \frac{1}{5}+\frac{1}{3}}}\|v\|_{X_{s_{1}, s_{2}}^{\frac{1}{2}+, \frac{1}{5}+, \frac{1}{3}}} .
$$

Remark 1.1. To prove Theorem 1.2, we make use of both the Strichartz-type inequalities and direct estimates for the kernel in the integral representation of the nonlinear estimate $23,33,34,37$. As in the case of the $\mathrm{KdV}$ equation and the KP-II equation, one of the difficulties comes from the derivative nonlinearity. We need to treat the small frequencies different from the high frequencies in the integral representation of the bilinear estimate. Also note that the extra factor

$$
1+\frac{\langle\tau-p(\xi, \eta)\rangle^{b_{1}}}{\langle\xi\rangle^{b_{2}}}
$$

in the definition of the Fourier transform restriction spaces $X_{s_{1}, s_{2}}^{b, b_{1}, b_{2}}$ is needed together with the Strichartz inequalities to treat the small frequencies in the proof of the crucial bilinear estimate. Another difficulty here concerns the Strichartz estimate. Unlike the KP-II case, we are not able to obtain a uniform-in-time Strichartz estimate for the linear problem of equation (1.1) due to the rotation term. Our approach follows the idea in 4, which is based on the study of the following oscillatory integral associated to the linear rmKP problem:

$$
I_{\alpha}(x, t, \beta, \gamma)=\int_{0}^{\infty} \xi^{\alpha} e^{i t(h(\xi)-x \xi)} d \xi, \quad t>0, x \in \mathbb{R},
$$

where $h(\xi)=-\beta \xi^{3}-\frac{\gamma}{\xi}$. The "long-time" estimate of the above oscillatory integral only gives a time-decay rate of $t^{-1 / 3}$ because, with the rotation term, $h^{\prime \prime}(\xi)$ has a zero at some nonzero $\xi$, which is different from the one of the amplitude function $\xi^{\alpha}$. Hence we cannot use the "cancellation effect" introduced in 23 to obtain the $t^{-1 / 2}$ decay. But the "short-time" estimate suggests a decay rate of $t^{-1 / 2}$, which is the same as that of the KP-II equation. However, if we split the space frequency into high frequency and low frequency parts, then we obtain the uniform time-decay estimates of the corresponding oscillatory integrals, and therefore the Strichartz estimates on both parts become uniform in time (see Lemma 2.6). Moreover, the time-decay estimate on the high frequency part is the same as that of the KP-II equation $\left(t^{-1 / 2}\right)$, which leads to the same result on the bilinear estimate as that of the KP-II equation. On the low frequency part the decay rate is $t^{-1 / 3}$, which seems 
to improve the blinear estimate. However, in the bilinear estimate, when dealing with the "high-low" frequency interaction, the rotation term generates some extra growth term than in the KP-II case, preventing us from getting the same lowregularity result as in KP-II.

A consequence of Theorem 1.3 is our local well-posedness result for the Cauchy problem (1.3). The method involves applying the Picard fixed point argument to the integral equation (1.8). Using the arguments of linear estimates in Bourgain's framework, we have the following theorem.

Theorem 1.4. Consider equation (1.1) with $\beta<0$ and $\gamma>0$. Let $s_{1}>-\frac{3}{10}$ and $s_{2} \geqslant 0$. Then for any $\phi \in H^{s_{1}, s_{2}}\left(\mathbb{R}^{2}\right)$ such that $|\xi|^{-1} \hat{\phi}(\xi, \eta) \in \mathcal{S}^{\prime}\left(\mathbb{R}^{2}\right)$ (the Schwartz class), there exist a positive $T=T\left(\|\phi\|_{H^{s_{1}, s_{2}}}\right)\left(\lim _{r \rightarrow 0} T(r)=\infty\right)$ and a unique solution $u(t, x, y)$ of the Cauchy problem (1.3) on the time interval $I=[-T, T]$ satisfying $u \in C\left(I, H^{s_{1}, s_{2}}\left(\mathbb{R}^{2}\right)\right) \cap X_{s_{1}, s_{2}}^{1 / 2+1 / 6+, 1 / 3}(I)$.

Remark 1.2. It was shown in [34 that $s_{1}=-1 / 3$ is the critical exponent for the bilinear estimate associated to the KP-II equation and hence to the local wellposedness of the KP-II equation in the anisotropic Sobolev space $H^{s_{1}, 0}\left(\mathbb{R}^{2}\right)$. As explained above, the rotation term improves the Strichartz estimate on the low frequency part and remains the same as KP-II on the high frequency part, but it also generates an extra growing factor in the bilinear estimate. Hence here we only obtained $s_{1}>-\frac{3}{10}$. One may expect the well-posedness for the rmKP equation with even smaller $s_{1}$. However, so far we have not been able to push $s_{1}$ further below $-\frac{3}{10}$ with $\beta<0$.

Formally, equation (1.3) has the following conservation laws:

$$
\begin{gathered}
E(u)=\int_{\mathbb{R}^{2}}\left(\frac{1}{2} \beta u_{x}^{2}+\frac{1}{3} u^{3}+\frac{1}{2}\left(\partial_{x}^{-1} u_{y}\right)^{2}+\frac{1}{2} \gamma\left(\partial_{x}^{-1} u\right)^{2}\right) d x d y, \\
V(u)=\frac{1}{2} \int_{\mathbb{R}^{2}} u^{2} d x d y,
\end{gathered}
$$

and

$$
M(u)=\int_{\mathbb{R}^{2}} u d x d y=0
$$

expressing, respectively, the energy, momentum and total mass ${ }^{*}$ Here, $\partial_{x}^{-1} u$ and $\partial_{x}^{-1} u_{y}$ are defined via the Fourier transform as

$$
\left(\partial_{x}^{-1} u\right)^{\wedge}(\xi, \eta)=\hat{u}(\xi, \eta) / i \xi \quad \text { and } \quad\left(\partial_{x}^{-1} u_{y}\right)^{\wedge}(\xi, \eta)=(\eta / \xi) \hat{u}(\xi, \eta) .
$$

A combination of Theorem 1.4 and the $L^{2}$-conservation law (1.14) yields the following global well-posedness theorem.

Theorem 1.5. Let $\beta<0$ and $\gamma>0$. Then for any $\phi \in L^{2}\left(\mathbb{R}^{2}\right)$ such that $|\xi|^{-1} \hat{\phi}(\xi, \eta) \in \mathcal{S}^{\prime}\left(\mathbb{R}^{2}\right)$, there exists a unique global solution $u$ of (1.3) satisfying $u \in C\left(\mathbb{R}, L^{2}\left(\mathbb{R}^{2}\right)\right)$.

*For a general class of Kadomtsev-Petviashvili type, it is proved [27 that the zero-mass in the $x$-variable

$$
\int_{-\infty}^{\infty} u(t ; x, y) d x=0
$$

holds for $t \in(0, T)$ even if it is not satisfied at $t=0$. 
In the second part of this paper we study the decay property of the solitary waves of equation (1.1).

A solitary wave of (1.1) is a traveling-wave solution of the form $\varphi(x-c t, y)$, where $\varphi \in X_{1}$ and $c \in \mathbb{R}$ is the speed of wave propagation. Equivalently, it is a solution $\varphi=\varphi(x, y)$ in $X_{1}$ of the equation

$$
-c \varphi_{x}-\beta \varphi_{x x x}+\left(\varphi^{2}\right)_{x}+\partial_{x}^{-1} \varphi_{y y}-\gamma \partial_{x}^{-1} \varphi=0
$$

for some $c \in \mathbb{R}$.

It has been shown in 10 that the solitary waves of equation (1.1) exist only when $\beta, \gamma>0$ and $c<2 \sqrt{\beta \gamma}$, which falls out of the case we discuss for the local well-posedness $(\beta<0, \gamma>0)$. In fact, when $\beta>0$, a "bad sign" in the identity $p(\xi, \eta)$ for the rmKP equation presents an apparent obstruction to performing the bilinear estimate.

In [10] it is proved that when $\beta, \gamma>0$ and $c<2 \sqrt{\beta \gamma}$, all solitary waves decay algebraically, which is no better than in the KP-I case [7. Here we obtain an improved result. We prove that such solitary waves decay exponentially at infinity. It can actually be viewed from the fact that the rotation term added to the kernel smooths out the singularity as in the KP case and hence improves the decay of the solutions. To be more precise, we have the following result.

Theorem 1.6. Let $\beta, c$ and $\gamma$ be constants satisfying $\beta, \gamma>0$ and $c<2 \sqrt{\beta \gamma}$. Then for any solitary wave $\varphi$ of (1.1), we have

$$
\varphi(x, y)=O\left(e^{-\alpha \sqrt{x^{2}+y^{2}}}\right),
$$

with $\alpha$ a positive constant depending only on $\beta, c$ and $\gamma$.

Remark 1.3. By (1.17), we obtain the exponential decay of solitary wave $\varphi$ with a positive number $\alpha$ provided $\beta, \gamma>0$ and $c<2 \sqrt{\beta \gamma}$, which definitely improves the algebraic decay estimate in Theorem 2.6 of [10], although in this paper we do not find out the optimal number $\alpha$ such that (1.17) holds for solitary waves.

To prove Theorem 1.6, we start with an integral decay estimate, which was used by Ambroseti, Felli and Malchiodi in proving integral decay results of the nonlinear Schrödinger equation (Lemma 17 of [3]). Then the use of an anisotropic Sobolev embedding theorem provides the $L^{\infty}$ bounds for the solitary waves.

This paper is organized as follows. In Section 2 we set up the Strichartz inequality for the rmKP equation (1.1) and use this to prove a crucial estimate Lemma 2.8 for the bilinear estimate. Section 3 is devoted to the proof of the bilinear estimate. In Section 4 we apply a Picard fixed-point argument to the integral equation (1.8), which implies the local well-posedness in some suitably chosen space. In Section 5 we show that all solitary waves obtained in 10] decay exponentially.

Notation. We denote by $\hat{\imath}$ or $\mathcal{F}$ the Fourier transform and by $\mathcal{F}^{-1}$ the inverse Fourier transform. $A \sim B$ means that there exists a constant $C \geqslant 1$ such that $|A| / C \leqslant|B| \leqslant C|A|$. The notation $a \pm$ means $a \pm \epsilon$ form arbitrarily small $\epsilon>0$. Constants are denoted by $C$ and may change from line to line.

Let $\zeta=(\xi, \eta), \zeta_{1}=\left(\xi_{1}, \eta_{1}\right)$ and let

$$
\begin{array}{lll}
\sigma:=\sigma(\tau, \zeta)=\tau-p(\xi, \eta), & \sigma_{1}:=\sigma\left(\tau_{1}, \zeta_{1}\right), & \sigma_{2}=\sigma\left(\tau-\tau_{1}, \zeta-\zeta_{1}\right), \\
\theta:=\theta(\tau, \zeta)=\frac{\langle\sigma\rangle^{b_{1}}}{\langle\xi\rangle^{b_{2}}}, & \theta_{1}:=\theta\left(\tau_{1}, \zeta_{1}\right), & \theta_{2}:=\theta\left(\tau-\tau_{1}, \zeta-\zeta_{1}\right) .
\end{array}
$$


Let the two projection operators $P_{N}$ and $P^{N}$ be defined as follows:

$$
\widehat{P_{N} f}(\xi, \eta)=\chi_{|\xi| \leqslant N} \hat{f}(\xi, \eta), \quad \widehat{P^{N} f}(\xi, \eta)=\chi_{|\xi| \geqslant N} \hat{f}(\xi, \eta) .
$$

\section{Linear estimates}

We first state the result on the estimate of the linear solution operator $S(t)$.

Theorem 2.1. Let $\beta<0, \gamma>0$ and $N=\left(\frac{2 \gamma}{-3 \beta}\right)^{1 / 4}$. If $\phi \in L^{1}\left(\mathbb{R}^{2}\right)$, then $S(t) \phi \in L^{\infty}\left(\mathbb{R}^{2}\right)$ and

$$
\begin{aligned}
& \left\|P_{N} S(t) \phi\right\|_{L^{\infty}\left(\mathbb{R}^{2}\right)} \leqslant C|t|^{-5 / 6}\|\phi\|_{L^{1}\left(\mathbb{R}^{2}\right)}, \\
& \left\|P^{N} S(t) \phi\right\|_{L^{\infty}\left(\mathbb{R}^{2}\right)} \leqslant C|t|^{-1}\|\phi\|_{L^{1}\left(\mathbb{R}^{2}\right)} .
\end{aligned}
$$

To prove this, we need the following lemma.

Lemma 2.2 (Van der Corput Lemma 31]). Suppose $\phi$ is real-valued and smooth in $(a, b)$ and that $\left|\phi^{(k)}\right| \geqslant c>0$ in $(a, b)$. Then

$$
\left|\int_{a}^{b} e^{i \lambda \phi(\xi)} \psi(\xi) d \xi\right| \leqslant C_{k}(c \lambda)^{-1 / k}\left[|\psi(b)|+\int_{a}^{b}\left|\psi^{\prime}(\xi)\right| d \xi\right]
$$

holds with $C_{k}$ independent of $\phi, \psi$ and $\lambda$ when (1) $k \geqslant 2$, or (2) $k=1$ and $\phi^{\prime}(\xi)$ is monotonic.

Applying the above lemma we obtain the "long-time" estimate.

Lemma 2.3. Let $\beta<0, \gamma>0, N=\left(\frac{2 \gamma}{-3 \beta}\right)^{1 / 4}$ and $0 \leqslant \alpha \leqslant 1 / 2$. Fix $\delta>0$. Define

$$
I_{\alpha}(x, t, \beta, \gamma)=\int_{0}^{\infty} \xi^{\alpha} e^{i t(h(\xi)-x \xi)} d \xi, \quad t>0, x \in \mathbb{R}
$$

where

Then

$$
h(\xi)=-\beta \xi^{3}-\frac{\gamma}{\xi}
$$

where $C=C(\alpha, \beta, \delta)$.

Proof. A simple computation shows that $\left|h^{\prime \prime \prime}(\xi)\right|=-6 \beta+\frac{6 \gamma}{\xi^{4}} \geqslant-6 \beta>0$. Hence from the Van der Corput Lemma we have

$$
\left|\int_{0}^{N+1} \xi^{\alpha} e^{i t(h(\xi)-x \xi)} d \xi\right| \leqslant C_{3}(-6 \beta)^{-1 / 3}(N+1)^{\alpha} t^{-1 / 3}, \quad t>0, x \in \mathbb{R},
$$

where $C_{3}$ is independent of $\alpha, \beta, \gamma, \delta$ and $t$.

We will argue as in [4. First we have that

$$
h^{\prime \prime}(\xi)=-6 \beta \xi-\frac{2 \gamma}{\xi^{3}} \geqslant-3 \beta \xi>0, \quad \text { for } \xi \geqslant N=\left(\frac{2 \gamma}{-3 \beta}\right)^{1 / 4} .
$$

We also have

$$
h^{\prime}(\xi)=-3 \beta \xi^{2}+\frac{\gamma}{\xi^{2}} \geqslant-3 \beta \xi^{2}>0 .
$$

Hence it follows that there exists at most one point $\xi_{x} \geqslant N+1$ such that $h^{\prime}\left(\xi_{x}\right)=x$. 
Define for $x \in \mathbb{R}$ the two sets

$$
A_{x}:=\left\{\xi \geqslant N+1:\left|\xi-\xi_{x}\right| \leqslant \frac{\xi_{x}}{2}\right\}, \quad B_{x}:=[N+1, \infty) \backslash A_{x} .
$$

(Note that $A_{x}$ can be empty.) Since $\frac{1}{2} \leqslant \frac{\xi}{\xi_{x}} \leqslant \frac{3}{2}$ in $A_{x}$, we may apply the Van der Corput Lemma to get

$$
\begin{aligned}
\left|\int_{A_{x}} \xi^{\alpha} e^{i t(h(\xi)-x \xi)} d \xi\right| & \leqslant C_{2} \xi_{x}^{\alpha}\left(-3 \beta \xi_{x} t\right)^{-1 / 2} \leqslant C_{2}(-3 \beta)^{-1 / 2}(N+1)^{\alpha-\frac{1}{2}} t^{-1 / 2} \\
& \leqslant C_{2}(-3 \beta)^{-1 / 2} t^{-1 / 2}
\end{aligned}
$$

where $C_{2}$ is independent of $\alpha, \beta, \gamma, \delta$ and $t$, provided $0 \leqslant \alpha \leqslant \frac{1}{2}$.

If $A_{x}$ is not empty, then for $\xi \in B_{x}$, we have either $N+1 \leqslant \xi<\frac{\xi_{x}}{2}$ or $\xi>\frac{3 \xi_{x}}{2}$. If $N+1 \leqslant \xi<\frac{\xi_{x}}{2}$, then

$$
\begin{aligned}
\left|h^{\prime}(\xi)-x\right| & =h^{\prime}\left(\xi_{x}\right)-h^{\prime}(\xi)=\int_{\xi}^{\xi_{x}} h^{\prime \prime}(\eta) d \eta \\
& \geqslant-\frac{3}{2} \beta\left(\xi_{x}+\xi\right)\left(\xi_{x}-\xi\right)>-\frac{9}{2} \beta \xi^{2} .
\end{aligned}
$$

If $\xi>\frac{3 \xi_{x}}{2}$, then

$$
\begin{aligned}
\left|h^{\prime}(\xi)-x\right| & =h^{\prime}(\xi)-h^{\prime}\left(\xi_{x}\right)=\int_{\xi_{x}}^{\xi} h^{\prime \prime}(\eta) d \eta \\
& \geqslant-\frac{3}{2} \beta\left(\xi+\xi_{x}\right)\left(\xi-\xi_{x}\right) \geqslant-\frac{1}{2} \beta \xi^{2} .
\end{aligned}
$$

In either case, we have

$$
\left|h^{\prime}(\xi)-x\right| \geqslant-\frac{1}{2} \beta \xi^{2}, \quad \xi \in B_{x} .
$$

If $A_{x}$ is empty (i.e. $B_{x}=[N+1, \infty)$ ), then the same estimate (2.8) holds for $\xi \geqslant \frac{3}{2}(N+1)$ since $h^{\prime}(N+1)>x$ and then $h^{\prime}(\xi)-x>h^{\prime}(\xi)-h^{\prime}(N+1)$. Therefore, replacing $B_{x}$ by $\left[\frac{3}{2}(N+1), \infty\right)$ if needed, and retaining the notation $B_{x}$, we obtain that (2.8) holds for all $x \in \mathbb{R}$. Thus we have

$$
\begin{aligned}
\mid \int_{B_{x}} & \xi^{\alpha} e^{i t(h(\xi)-x \xi)} d \xi \mid \\
& =\left|\frac{1}{i t} \int_{B_{x}} \frac{\xi^{\alpha}}{h^{\prime}(\xi)-x}\left(\frac{d}{d \xi} e^{i t(h(\xi)-x \xi)}\right) d \xi\right| \\
& \leqslant t^{-1}\left\{\sup _{\xi \in B_{x}} \frac{\xi^{\alpha}}{\left|h^{\prime}(\xi)-x\right|}+\int_{B_{x}}\left|\frac{d}{d \xi} \frac{\xi^{\alpha}}{h^{\prime}(\xi)-x}\right| d \xi\right\} \\
& \leqslant t^{-1}\left\{-\frac{2}{\beta}(N+1)^{\alpha-2}+\int_{B_{x}} \frac{\left|\alpha \xi^{\alpha-1}\left(h^{\prime}(\xi)-x\right)-\xi^{\alpha} h^{\prime \prime}(\xi)\right|}{\left|h^{\prime}(\xi)-x\right|^{2}} d \xi\right\} \\
& \leqslant t^{-1}\left\{-\frac{2}{\beta}(N+1)^{\alpha-2}+\int_{B_{x}}-\frac{2 \alpha+24}{\beta} \xi^{\alpha-3} d \xi\right\} \\
& \leqslant \frac{4 \alpha+20}{\beta(\alpha-2)}(N+1)^{\alpha-2} t^{-1} \leqslant \frac{4 \alpha+20}{\beta(\alpha-2)} t^{-1},
\end{aligned}
$$

when $\alpha<2$.

Combining (2.6), (2.7) and (2.9) we obtain (2.5). 
Moreover, we have the "short-time" estimate.

Lemma 2.4. Let $\beta<0, \gamma>0$ and $0 \leqslant \alpha \leqslant 1 / 2$. Fix $T>0$. Then

$$
\sup _{x \in \mathbb{R}}\left|I_{\alpha}(x, t, \beta, \gamma)\right| \leqslant C t^{-\frac{\alpha+1}{3}}, \quad 0<t \leqslant T
$$

where $C=C(\alpha, \beta, \gamma, T)$.

Proof. Performing the change of variable $\xi=\lambda \eta$ with $\lambda \geqslant 1$, we have

$$
\begin{aligned}
I_{\alpha}(x, t, \beta, \gamma) & =\lambda^{\alpha+1} \int_{0}^{\infty} \eta^{\alpha} e^{i\left(\lambda^{3} t\right)\left[-\beta \eta^{3}-\frac{\lambda^{-4} \gamma}{\eta}-\left(\lambda^{-2} x\right) \eta\right]} d \eta \\
& =\lambda^{\alpha+1} I_{\alpha}\left(\lambda^{-2} x, \lambda^{3} t, \beta, \lambda^{-4} \gamma\right) .
\end{aligned}
$$

Hence

$\sup _{x \in \mathbb{R}}\left|I_{\alpha}(x, t, \beta, \gamma)\right| \leqslant \lambda^{\alpha+1} \sup _{x \in \mathbb{R}}\left|I_{\alpha}\left(\lambda^{-2} x, \lambda^{3} t, \beta, \lambda^{-4} \gamma\right)\right| \leqslant \lambda^{\alpha+1} \sup _{x \in \mathbb{R}}\left|I_{\alpha}\left(x, \lambda^{3} t, \beta, \gamma\right)\right|$, where in the last inequality we have used the estimate (2.5) and the assumption that $\lambda \geqslant 1$.

Therefore, for $0<t \leqslant T$, setting $\lambda=(T / t)^{1 / 3}$ we obtain (2.10).

Proof of Theorem 2.1. Consider $\xi$ being positive (the negative case can be treated the same way). Making the change of variable $\rho=(|t| / \xi)^{1 / 2} \eta$ we have

$$
\begin{aligned}
S(t)^{+} & =\int_{\mathbb{R}} \int_{0}^{\infty} e^{i t\left[-\beta \xi^{3}-\left(\eta^{2}+\gamma\right) / \xi\right]} e^{i(x \xi+y \eta)} d \xi d \eta \\
& =|t|^{-1 / 2} \int_{0}^{\infty} \xi^{1 / 2} e^{i\left[\left(x-y^{2} /|t|\right) \xi-t\left(\beta \xi^{3}+\gamma / \xi\right)\right]} d \xi .
\end{aligned}
$$

Then (2.1) can be obtained from (2.6).

As for (2.2), from (2.7) and (2.9) with $\alpha=1 / 2$ we see that

$$
\sup _{x \in \mathbb{R}}\left|\int_{N}^{\infty} \xi^{1 / 2} e^{i t(h-x \xi)} d \xi\right| \leqslant C t^{-1 / 2}, \text { for } t>\delta>0,
$$

where $C=C(\beta, \gamma, N, \delta)$. Furthermore, from Lemma 2.4 we have for $0<t \leqslant \delta$

$$
\begin{aligned}
C t^{-1 / 2} & \geqslant \sup _{x \in \mathbb{R}}\left|\int_{0}^{\infty} \xi^{1 / 2} e^{i t(h-x \xi)} d \xi\right| \\
& \geqslant \sup _{x \in \mathbb{R}}\left|\int_{N}^{\infty} \xi^{1 / 2} e^{i t(h-x \xi)} d \xi\right|-\sup _{x \in \mathbb{R}}\left|\int_{0}^{N} \xi^{1 / 2} e^{i t(h-x \xi)} d \xi\right| .
\end{aligned}
$$

Hence

$$
\begin{aligned}
\sup _{x \in \mathbb{R}}\left|\int_{N}^{\infty} \xi^{1 / 2} e^{i t(h-x \xi)} d \xi\right| & \leqslant C t^{-1 / 2}+\sup _{x \in \mathbb{R}}\left|\int_{0}^{N} \xi^{1 / 2} e^{i t(h-x \xi)} d \xi\right| \\
& \leqslant C\left(t^{-1 / 2}+t^{-1 / 3}\right) \leqslant C t^{-1 / 2}
\end{aligned}
$$

if $\delta<1$. Hence we obtain that

$$
\sup _{x \in \mathbb{R}}\left|\int_{N}^{\infty} \xi^{1 / 2} e^{i t(h-x \xi)} d \xi\right| \leqslant C t^{-1 / 2}
$$

Therefore we have proved (2.2). 
As a consequence of Theorem 2.1 we have

Proposition 2.5. Let $\beta<0, \gamma>0$ and $N=\left(\frac{2 \gamma}{-3 \beta}\right)^{1 / 4}$. For any $\theta \in[0,1]$ and $\phi \in \partial_{x}\left(C_{0}^{\infty}\left(\mathbb{R}^{2}\right)\right)$,

$$
\begin{aligned}
& \left\|P_{N} S(t) \phi\right\|_{L^{2 /(1-\theta)}} \leqslant C|t|^{-\frac{5 \theta}{6}}\|\phi\|_{L^{2 /(1+\theta)}}, \\
& \left\|P^{N} S(t) \phi\right\|_{L^{2 /(1-\theta)}} \leqslant C|t|^{-\theta}\|\phi\|_{L^{2 /(1+\theta)} .}
\end{aligned}
$$

Proof. As in [23], we introduce, for $\lambda \in[0,1], \mu \in \mathbb{R}$, the analytic family of operators

$$
W_{\lambda+i \mu}(t) \phi(x, y)=\int_{\mathbb{R}^{2}}|\xi|^{(\lambda+i \mu) / 2} e^{i t p(\xi, \eta)+i(x \xi+y \eta)} \hat{\phi}(\xi, \eta) d \xi d \eta .
$$

A slight modification of Theorem 2.1 implies that

$$
\begin{aligned}
& \left\|P_{N} W_{1+i \mu}(t) \phi\right\|_{L^{\infty}} \leqslant C(1+|\lambda|)|t|^{-5 / 6}\|\phi\|_{L^{1}}, \\
& \left\|P^{N} W_{1+i \mu}(t) \phi\right\|_{L^{\infty}} \leqslant C(1+|\lambda|)|t|^{-1}\|\phi\|_{L^{1}},
\end{aligned}
$$

while by unitary,

$$
\left\|W_{i \mu}(t) \phi\right\|_{L^{2}}=\left\|D^{i \lambda} \phi\right\|_{L^{2}}=\|\phi\|_{L^{2}} .
$$

Hence by complex interpolation (see 32 Chapter V, Theorem 4.1) we obtain (2.11) and (2.12).

It is standard now (see for instance [23]) to deduce the following "Strichartz estimates".

Lemma 2.6. Let $\beta<0, \gamma>0$ and $N=\left(\frac{2 \gamma}{-3 \beta}\right)^{1 / 4}$. For any $\theta \in[0,1]$

$$
\begin{aligned}
& \left\|P_{N} S(t) \phi\right\|_{L^{q_{1}}\left(\mathbb{R} ; L^{p_{1}}\left(\mathbb{R}^{2}\right)\right)} \leqslant C\|\phi\|_{L^{2}}, \\
& \left\|P^{N} S(t) \phi\right\|_{L^{q_{2}}\left(\mathbb{R} ; L^{p_{2}}\left(\mathbb{R}^{2}\right)\right)} \leqslant C\|\phi\|_{L^{2}},
\end{aligned}
$$

where $\left(q_{1}, p_{1}\right)=\left(\frac{12}{5 \theta}, \frac{2}{1-\theta}\right),\left(q_{2}, p_{2}\right)=\left(\frac{2}{\theta}, \frac{2}{1-\theta}\right)$.

Proof. We will just prove (2.13). The same argument applies to the proof of (2.14).

By duality, (2.13) is equivalent to

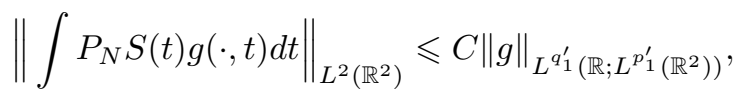

where $1 / q_{1}+1 / q_{1}^{\prime}=1,1 / p_{1}+1 / p_{1}^{\prime}=1$.

By the argument in 36 .

$$
\begin{aligned}
\left\|\int P_{N} S(t) g(\cdot, t) d t\right\|_{L^{2}\left(\mathbb{R}^{2}\right)}^{2} & =\int\left(\int P_{N} S(t) g(\cdot, t) d t\right)\left(\int \overline{P_{N} S(t) g(\cdot, t) d t}\right) d x \\
& =\iint g(x, t)\left(\int P_{N} S(t-\tau) \overline{g(\cdot, \tau)} d \tau\right) d x d t .
\end{aligned}
$$

Therefore (2.13) is equivalent to

$$
\left\|\int P_{N} S(t-\tau) g(\cdot, \tau) d \tau\right\|_{L^{q_{1}\left(\mathbb{R} ; L^{p_{1}}\left(\mathbb{R}^{2}\right)\right)}} \leqslant C\|g\|_{L^{q_{1}^{\prime}\left(\mathbb{R} ; L^{p_{1}^{\prime}}\left(\mathbb{R}^{2}\right)\right)}} .
$$


We have from (2.11) that

$$
\begin{aligned}
& \left\|\int P_{N} S(t-\tau) g(\cdot, \tau) d \tau\right\|_{L^{q_{1}\left(\mathbb{R} ; L^{p_{1}}\left(\mathbb{R}^{2}\right)\right)}} \\
& \quad \leqslant\left\|\int\right\| P_{N} S(t-\tau) g(\cdot, \tau)\left\|_{L^{p_{1}\left(\mathbb{R}^{2}\right)}} d \tau\right\|_{L^{q_{1}(\mathbb{R})}} \\
& \quad \leqslant C\left\|\int|t-\tau|^{-5 \theta / 6}\right\| g(\cdot, \tau)\left\|_{L^{p_{1}^{\prime}\left(\mathbb{R}^{2}\right)}} d \tau\right\|_{L^{q_{1}(\mathbb{R})}} \\
& \quad \leqslant C\|g\|_{L^{q^{\prime}}\left(\mathbb{R} ; L^{p^{\prime}}\left(\mathbb{R}^{2}\right)\right)},
\end{aligned}
$$

where $p_{1}=2 /(1-\theta), 1 / q_{1}=1 / q_{1}^{\prime}-\left(1-\frac{5}{6} \theta\right)$, i.e. $q_{1}=12 /(5 \theta)$.

Lemma 2.7. Let $\beta<0, \gamma>0$ and $N=\left(\frac{2 \gamma}{-3 \beta}\right)^{1 / 4}$. For $2 \leqslant q_{1} \leqslant 22 / 5,2 \leqslant q_{2} \leqslant 4$ and any $u \in L^{2}\left(\mathbb{R}^{3}\right)$ we have

$$
\begin{aligned}
& \left\|P_{N} \mathcal{F}^{-1}\left(\langle\sigma\rangle^{-b_{1}}|\hat{u}(\tau, \zeta)|\right)\right\|_{L^{q_{1}}} \leqslant C\|u\|_{L^{2}}, \\
& \left\|P^{N} \mathcal{F}^{-1}\left(\langle\sigma\rangle^{-b_{2}}|\hat{u}(\tau, \zeta)|\right)\right\|_{L^{q_{2}}} \leqslant C\|u\|_{L^{2}}
\end{aligned}
$$

where

$$
b_{1}=\frac{11}{6}(1-2 / q)(1 / 2+), b_{2}=2(1-2 / q)(1 / 2+)
$$

and $\mathcal{F}^{-1}$ denotes the inverse Fourier transform.

Proof. From (2.13), taking $\theta=6 / 11$, we obtain for any $\phi \in L^{2}\left(\mathbb{R}^{2}\right)$

$$
\left\|P_{N} S(t) \phi\right\|_{L^{22 / 5}\left(\mathbb{R}^{3}\right)} \leqslant C\|\phi\|_{L^{2}\left(\mathbb{R}^{2}\right)} .
$$

From (2.17), [13, Lemma 3.3] gives, for any $u \in L^{2}\left(\mathbb{R}^{3}\right)$,

$$
\left\|P_{N} \mathcal{F}^{-1}\left(\langle\sigma\rangle^{-\frac{1}{2}-}|\hat{u}(\tau, \zeta)|\right)\right\|_{L^{22 / 5}} \leqslant C\|u\|_{L^{2}} .
$$

Interpolating (2.18) and the Plancherel identity we obtain (2.15). Similarly, (2.16) can be obtained by interpolating between

$$
\left\|P^{N} \mathcal{F}^{-1}\left(\langle\sigma\rangle^{-\frac{1}{2}-}|\hat{u}(\tau, \zeta)|\right)\right\|_{L^{4}} \leqslant C\|u\|_{L^{2}}
$$

and the Plancherel identity.

Lemma 2.8. Let $\beta<0, \gamma>0, a_{1}, a_{2}, a_{3} \in\left[0, \frac{1}{2}+\epsilon\right]$, and let $\hat{u_{1}}, \hat{u_{2}}, \hat{u_{3}}$ be positive. Then

$$
\int_{\mathbb{R}^{6}} \frac{\hat{u}_{1}\left(\tau_{1}, \zeta_{1}\right) \hat{u}_{2}\left(\tau-\tau_{1}, \zeta-\zeta_{1}\right) \hat{u}_{3}(\tau, \zeta)}{\left\langle\sigma_{1}\right\rangle^{a_{1}}\left\langle\sigma_{2}\right\rangle^{a_{2}}\langle\sigma\rangle^{a_{3}}} d \tau d \zeta d \tau_{1} d \zeta_{1} \leqslant C\|u\|_{L^{2}}\|v\|_{L^{2}}\|w\|_{L^{2}}
$$
provided $a_{1}+a_{2}+a_{3} \geqslant 1+2 \epsilon$.

Proof. We denote by $I$ the left-hand side of (2.19). Let $N=\left(\frac{2 \gamma}{-3 \beta}\right)^{1 / 4}$. Then we can rewrite $I$ as

$$
\begin{aligned}
I= & \int_{\mathbb{R}^{6}} \frac{\mathcal{F}\left(\left(P_{N}+P^{N}\right) u_{1}\right)\left(\tau_{1}, \zeta_{1}\right) \mathcal{F}\left(\left(P_{N}+P^{N}\right) u_{2}\right)\left(\tau-\tau_{1}, \zeta-\zeta_{1}\right) \mathcal{F}\left(\left(P_{N}+P^{N}\right) u_{3}\right)(\tau, \zeta)}{\left\langle\sigma_{1}\right\rangle^{a_{1}}\left\langle\sigma_{2}\right\rangle^{a_{2}}\langle\sigma\rangle^{a_{3}}} \\
& \times d \tau d \zeta d \tau_{1} d \zeta_{1} \\
= & \int_{\mathbb{R}^{6}} \frac{\sum_{i}\left(\text { products of } \mathcal{F}\left(P_{N} u_{i}\right) \text { and } \mathcal{F}\left(P^{N} u_{i}\right)\right)}{\left\langle\sigma_{1}\right\rangle^{a_{1}}\left\langle\sigma_{2}\right\rangle^{a_{2}}\langle\sigma\rangle^{a_{3}}} d \tau d \zeta d \tau_{1} d \zeta_{1} .
\end{aligned}
$$


By symmetry, we only need to estimate the following four terms:

$$
\begin{aligned}
I_{1} & =\int_{\mathbb{R}^{6}} \frac{\mathcal{F}\left(P_{N} u_{1}\right) \mathcal{F}\left(P_{N} u_{2}\right) \mathcal{F}\left(P_{N} u_{3}\right)}{\left\langle\sigma_{1}\right\rangle^{a_{1}}\left\langle\sigma_{2}\right\rangle^{a_{2}}\langle\sigma\rangle^{a_{3}}} d \tau d \zeta d \tau_{1} d \zeta_{1}, \\
I_{2} & =\int_{\mathbb{R}^{6}} \frac{\mathcal{F}\left(P^{N} u_{1}\right) \mathcal{F}\left(P_{N} u_{2}\right) \mathcal{F}\left(P_{N} u_{3}\right)}{\left\langle\sigma_{1}\right\rangle^{a_{1}}\left\langle\sigma_{2}\right\rangle^{a_{2}}\langle\sigma\rangle^{a_{3}}} d \tau d \zeta d \tau_{1} d \zeta_{1}, \\
I_{3} & =\int_{\mathbb{R}^{6}} \frac{\mathcal{F}\left(P^{N} u_{1}\right) \mathcal{F}\left(P^{N} u_{2}\right) \mathcal{F}\left(P_{N} u_{3}\right)}{\left\langle\sigma_{1}\right\rangle^{a_{1}}\left\langle\sigma_{2}\right\rangle^{a_{2}}\langle\sigma\rangle^{a_{3}}} d \tau d \zeta d \tau_{1} d \zeta_{1}, \\
I_{4} & =\int_{\mathbb{R}^{6}} \frac{\mathcal{F}\left(P^{N} u_{1}\right) \mathcal{F}\left(P^{N} u_{2}\right) \mathcal{F}\left(P^{N} u_{3}\right)}{\left\langle\sigma_{1}\right\rangle^{a_{1}}\left\langle\sigma_{2}\right\rangle^{a_{2}}\langle\sigma\rangle^{a_{3}}} d \tau d \zeta d \tau_{1} d \zeta_{1} .
\end{aligned}
$$

Using Hölder's inequality, Plancherel's identity, and Lemma 2.7 we obtain

$$
\begin{aligned}
I_{1} & \leqslant C\left\|P_{N} \mathcal{F}^{-1}\left(\left\langle\sigma_{1}\right\rangle^{-a_{1}}\left|\hat{u}_{1}\right|\right)\right\|_{L^{q_{1}}}\left\|P_{N} \mathcal{F}^{-1}\left(\left\langle\sigma_{2}\right\rangle^{-a_{2}}\left|\hat{u}_{2}\right|\right)\right\|_{L^{q_{2}}}\left\|P_{N} \mathcal{F}^{-1}\left(\langle\sigma\rangle^{-a_{3}}\left|\hat{u}_{3}\right|\right)\right\|_{L^{q_{3}}} \\
& \leqslant C\|u\|_{L^{2}}\|v\|_{L^{2}}\|w\|_{L^{2}},
\end{aligned}
$$

provided $a_{j}=\frac{11}{6}\left(1-2 / q_{j}\right)(1 / 2+)$ and $1 / q_{1}+1 / q_{2}+1 / q_{3}=1$. The last condition is equivalent to $a_{1}+a_{2}+a_{3}=\frac{11}{12}+2 \epsilon<1+2 \epsilon$.

As for $I_{4}$, we have

$$
\begin{aligned}
I_{4} & \leqslant C\left\|P^{N} \mathcal{F}^{-1}\left(\left\langle\sigma_{1}\right\rangle^{-a_{1}}\left|\hat{u}_{1}\right|\right)\right\|_{L^{q_{1}}}\left\|P^{N} \mathcal{F}^{-1}\left(\left\langle\sigma_{2}\right\rangle^{-a_{2}}\left|\hat{u}_{2}\right|\right)\right\|_{L^{q_{2}}}\left\|P^{N} \mathcal{F}^{-1}\left(\langle\sigma\rangle^{-a_{3}}\left|\hat{u}_{3}\right|\right)\right\|_{L^{q_{3}}} \\
& \leqslant C\|u\|_{L^{2}}\|v\|_{L^{2}}\|w\|_{L^{2}},
\end{aligned}
$$

provided $a_{j}=2\left(1-2 / q_{j}\right)(1 / 2+)$ and $1 / q_{1}+1 / q_{2}+1 / q_{3}=1$. The last condition is equivalent to $a_{1}+a_{2}+a_{3}=1+2 \epsilon$.

Using the same method for $I_{2}$ and $I_{3}$ we see that we need $\frac{11}{12}+2 \epsilon<a_{1}+a_{2}+a_{3}<$ $1+2 \epsilon$. But clearly by looking at $I$ we know that the larger $a_{1}+a_{2}+a_{3}$ is, the smaller the integrand becomes. Hence we only need to determine the lowest possible number that $a_{1}+a_{2}+a_{3}$ can attain. Therefore we can conclude that $a_{1}+a_{2}+a_{3} \geqslant 1+2 \epsilon$.

The following two elementary calculus inequalities are also useful in our proof of the bilinear estimates.

Lemma 2.9. For any $a \in \mathbb{R}$ the following inequalities hold:

$$
\begin{gathered}
\int_{-\infty}^{\infty} \frac{d t}{\langle t\rangle^{1 \pm}\langle t-a\rangle^{1+}} \leqslant \frac{C}{\langle a\rangle^{1 \pm}} \\
\int_{-\infty}^{\infty} \frac{d t}{\langle t\rangle^{1+}|t-a|^{1 / 2}} \leqslant \frac{C}{\langle a\rangle^{1 / 2}} .
\end{gathered}
$$

\section{Bilinear estimates}

In this section we prove the crucial bilinear estimate Theorem 1.2 .

Proof of Theorem 1.2. A duality argument shows that (1.9) is equivalent to

$$
\begin{aligned}
& \iint K\left(\tau, \zeta, \tau_{1}, \zeta_{1}\right) \hat{u}\left(\tau_{1}, \zeta_{1}\right) \hat{v}\left(\tau-\tau_{1}, \zeta-\zeta_{1}\right) \hat{w}(\tau, \zeta) d \tau_{1} d \zeta_{1} d \tau d \zeta \\
& \leqslant C\|u\|_{L^{2}}\|v\|_{L^{2}}\|w\|_{L^{2}},
\end{aligned}
$$

where $\hat{u}, \hat{v}, \hat{w}$ can be assumed to be positive and

$$
K\left(\tau, \zeta, \tau_{1}, \zeta_{1}\right)=\frac{|\xi|\langle\theta\rangle\langle\xi\rangle^{s_{1}}\left\langle\xi_{1}\right\rangle^{-s_{1}}\left\langle\xi-\xi_{1}\right\rangle^{-s_{1}}}{\langle\sigma\rangle^{b^{\prime}}\left\langle\sigma_{1}\right\rangle^{b}\left\langle\sigma_{2}\right\rangle^{b}\left\langle\theta_{1}\right\rangle\left\langle\theta_{2}\right\rangle} \frac{\langle\eta\rangle^{s_{2}}}{\left\langle\eta_{1}\right\rangle^{s_{2}}\left\langle\eta-\eta_{1}\right\rangle^{s_{2}}} .
$$


We consider only the case $-1 / 3<s_{1} \leqslant 0$ and denote $s=-s_{1}$. Moreover, from the inequality

$$
\frac{\langle\eta\rangle^{s_{2}}}{\left\langle\eta_{1}\right\rangle^{s_{2}}\left\langle\eta-\eta_{1}\right\rangle^{s_{2}}} \leqslant C
$$

we may just take the case $s_{2}=0$. Therefore the kernel $K\left(\tau, \zeta, \tau_{1}, \zeta_{1}\right)$ in (3.1) becomes

$$
K\left(\tau, \zeta, \tau_{1}, \zeta_{1}\right)=\frac{|\xi|\langle\theta\rangle\langle\xi\rangle^{-s}\left\langle\xi_{1}\right\rangle^{s}\left\langle\xi-\xi_{1}\right\rangle^{s}}{\langle\sigma\rangle^{b^{\prime}}\left\langle\sigma_{1}\right\rangle^{b}\left\langle\sigma_{2}\right\rangle^{b}\left\langle\theta_{1}\right\rangle\left\langle\theta_{2}\right\rangle} .
$$

We also have the relation

$$
\sigma_{1}+\sigma_{2}-\sigma=-3 \beta \xi \xi_{1}\left(\xi-\xi_{1}\right)+\frac{\left(\xi_{1} \eta-\xi \eta_{1}\right)^{2}}{\xi \xi_{1}\left(\xi-\xi_{1}\right)}+\gamma \frac{\xi^{2}-\xi \xi_{1}+\xi_{1}^{2}}{\xi \xi_{1}\left(\xi-\xi_{1}\right)}
$$

and therefore (cf. [8])

$$
\max \left\{|\sigma|,\left|\sigma_{1}\right|,\left|\sigma_{2}\right|\right\} \geqslant-\beta\left|\xi \xi_{1}\left(\xi-\xi_{1}\right)\right| .
$$

By symmetry we may assume that $\left|\sigma_{1}\right| \geqslant\left|\sigma_{2}\right|$. Denote by $J$ the left-hand side of (3.1). We break our argument into several cases.

Case 1: $|\xi|$ is small, say $|\xi| \leqslant 8$.

Case 1.1: $\left|\xi_{1}\right| \leqslant 1$. Denote by $J_{11}$ the contribution of the region to $J$. In this case, $\left|\xi-\xi_{1}\right| \leqslant 2$ and

$$
K\left(\tau, \zeta, \tau_{1}, \zeta_{1}\right) \leqslant \frac{C}{\langle\sigma\rangle^{b^{\prime}-b_{1}}\left\langle\sigma_{1}\right\rangle^{b}\left\langle\sigma_{2}\right\rangle^{b}} .
$$

Since $b^{\prime}-b_{1}>0, b>\frac{1}{2}$, the use of Lemma 2.8 implies the right bound for $J_{11}$.

Case 1.2: $\left|\xi_{1}\right| \geqslant 1$. In this case $\langle\xi\rangle^{-s}\left\langle\xi_{1}\right\rangle^{s}\left\langle\xi-\xi_{1}\right\rangle^{s} \leqslant C\left\langle\xi_{1}\right\rangle^{2 s}$. Denote by $J_{12}$ the contribution of the region to $J$.

- $\left|\sigma_{1}\right| \geqslant|\sigma|$ : From (3.2) we have $|\xi|^{s}\left\langle\xi_{1}\right\rangle^{2 s} \leqslant C\left\langle\sigma_{1}\right\rangle^{s}$. Since $\langle\theta\rangle \leqslant C\langle\sigma\rangle^{b_{1}}$, we know

$$
K\left(\tau, \zeta, \tau_{1}, \zeta_{1}\right) \leqslant \frac{C|\xi|^{1-s}}{\langle\sigma\rangle^{b^{\prime}-b_{1}}\left\langle\sigma_{1}\right\rangle^{b-s}\left\langle\sigma_{2}\right\rangle^{b}} .
$$

To apply Lemma 2.8, we need $b^{\prime}-b_{1}+b-s+b>1$. This can be seen from the fact that $b>\frac{1}{2}$ and $b^{\prime}-b_{1}>s$.

- $|\sigma| \geqslant\left|\sigma_{1}\right|$ : From (3.2) we have $|\xi|^{s}\left\langle\xi_{1}\right\rangle^{2 s} \leqslant C\langle\sigma\rangle^{s}$. Hence

$$
K\left(\tau, \zeta, \tau_{1}, \zeta_{1}\right) \leqslant \frac{C|\xi|^{1-s}}{\langle\sigma\rangle^{b^{\prime}-b_{1}-s}\left\langle\sigma_{1}\right\rangle^{b}\left\langle\sigma_{2}\right\rangle^{b}} .
$$

Since $b>\frac{1}{2}$ and $b^{\prime}-b_{1}>s$, we know that $b^{\prime}-b_{1}-s+b+b>1$. So we can use Lemma 2.8 to get the right bound for $J_{12}$.

Case 2: $|\xi| \geqslant 8$.

Case 2.1: $|\sigma| \geqslant\left|\sigma_{1}\right|$ and $\min \left\{\left|\xi_{1}\right|,\left|\xi-\xi_{1}\right|\right\} \geqslant 1$.

Case 2.1.1: $|\xi| \leqslant 10 \min \left\{\left|\xi_{1}\right|,\left|\xi-\xi_{1}\right|\right\}$. Denote by $J_{211}$ the contribution of this region to $J$. Using (3.2) we know that on the support of $J_{211}$,

$$
|\xi|^{1-s}\left|\xi_{1}\right|^{s}\left|\xi-\xi_{1}\right|^{s} \leqslant C\left|\xi \xi_{1}\left(\xi-\xi_{1}\right)\right|^{(1+s) / 3} \leqslant C\langle\sigma\rangle^{(1+s) / 3} .
$$


- If $\langle\sigma\rangle^{b_{1}} \leqslant|\xi|^{b_{2}}$, then

$$
K\left(\tau, \zeta, \tau_{1}, \zeta_{1}\right) \leqslant \frac{C}{\langle\sigma\rangle^{b^{\prime}-\frac{1+s}{3}}\left\langle\sigma_{1}\right\rangle^{b}\left\langle\sigma_{2}\right\rangle^{b}} .
$$

Since $b^{\prime}-\frac{1+s}{3}>0$, we can use Lemma 2.8 ,

- If $\langle\sigma\rangle^{b_{1}} \geqslant|\xi|^{b_{2}}$, then

$$
K\left(\tau, \zeta, \tau_{1}, \zeta_{1}\right) \leqslant \frac{|\xi|^{1-s-b_{2}}\left|\xi_{1}\right|^{s}\left|\xi-\xi_{1}\right|^{s}}{\langle\sigma\rangle^{b^{\prime}-b_{1}}\left\langle\sigma_{1}\right\rangle^{b}\left\langle\sigma_{2}\right\rangle^{b}} \leqslant \frac{C}{\langle\sigma\rangle^{b^{\prime}-b_{1}-\max \left\{s, \frac{1+s-b_{2}}{3}\right\}}\left\langle\sigma_{1}\right\rangle^{b}\left\langle\sigma_{2}\right\rangle^{b}} .
$$

Since $b^{\prime}-b_{1}>s$ and $b^{\prime}-b_{1}>\frac{1+s-b_{2}}{3}$, we may use Lemma 2.8 to complete the proof in this case.

Case 2.1.2: $|\xi| \geqslant 10 \min \left\{\left|\xi_{1}\right|,\left|\xi-\xi_{1}\right|\right\}$. Denote by $J_{212}$ the contribution of this region to $J$. On the support of $J_{212}$ we have

$$
|\xi|^{1-s}\left|\xi_{1}\right|^{s}\left|\xi-\xi_{1}\right|^{s} \leqslant C|\xi|^{1+s}
$$

Using the Cauchy-Schwarz inequality, we obtain

$$
J_{212} \leqslant C \int I(\tau, \zeta)\left\{\int\left|\hat{u}\left(\tau_{1}, \zeta_{1}\right) \hat{v}\left(\tau-\tau_{1}, \zeta-\zeta_{1}\right)\right|^{2} d \tau_{1} d \zeta_{1}\right\}^{1 / 2} \hat{w}(\tau, \zeta) d \tau d \zeta,
$$

where

$$
I(\tau, \zeta)=\frac{|\xi|^{1+s}\langle\theta\rangle}{\langle\sigma\rangle^{b^{\prime}}}\left(\int_{|\sigma| \geqslant\left|\sigma_{1}\right|} \frac{d \tau_{1} d \zeta_{1}}{\left\langle\sigma_{1}\right\rangle^{2 b}\left\langle\sigma_{2}\right\rangle^{2 b}\left\langle\theta_{1}\right\rangle^{2}\left\langle\theta_{2}\right\rangle^{2}}\right)^{1 / 2} .
$$

We will show that $I(\tau, \zeta)$ is bounded for $|\xi| \geqslant 1$. Using (2.20) we have

$$
I(\tau, \zeta) \leqslant \frac{C|\xi|^{1+s}\langle\theta\rangle}{\langle\sigma\rangle^{b^{\prime}}}\left(\int \frac{d \xi_{1} d \eta_{1}}{\left\langle\sigma_{1}+\sigma_{2}\right\rangle^{2 b}}\right)^{1 / 2} .
$$

We perform the change of variables $\left(\xi_{1}, \eta_{1}\right) \mapsto(\nu, \mu)$ :

$$
\nu=-3 \beta \xi \xi_{1}\left(\xi-\xi_{1}\right), \quad \mu=\sigma_{1}+\sigma_{2} .
$$

Hence

$$
\begin{aligned}
& \nu \in\left[-3|\sigma|, \min \left\{-\frac{3 \beta}{4} \xi^{3}, 3|\sigma|\right\}\right], \quad \text { when } \xi \geqslant 0, \\
& \nu \in\left[\max \left\{-\frac{3 \beta}{4} \xi^{3},-3|\sigma|\right\}, 3|\sigma|\right], \quad \text { when } \xi \leqslant 0 .
\end{aligned}
$$

We will discuss the case $\xi \geqslant 0$. The case $\xi \leqslant 0$ can be treated the same way. In this case we have

$$
d \xi_{1} d \eta_{1}=\frac{|\nu|^{1 / 2} d \nu d \mu}{12(-\beta)^{3 / 2}|\xi|^{3 / 2} \sqrt{\frac{3}{4} \xi^{3}+\frac{1}{\beta} \nu}|\sigma+\nu+k-\mu|^{1 / 2}},
$$

where

$$
k=\frac{-3 \beta \gamma\left(\xi^{2}-\xi \xi_{1}+\xi_{1}^{2}\right)}{\nu}=\frac{A}{\nu},
$$

where we denote

$$
A=-3 \beta \gamma\left(\xi^{2}-\xi \xi_{1}+\xi_{1}^{2}\right) .
$$


Thus using (2.21) we obtain

$$
\begin{aligned}
I(\tau, \zeta) \leqslant & \frac{C|\xi|^{\frac{1}{4}+s}\langle\theta\rangle}{\langle\sigma\rangle^{b^{\prime}}} \\
& \times\left\{\int_{-3|\sigma|}^{3|\sigma|} \int_{-\infty}^{\infty} \frac{|\nu|^{1 / 2} d \mu d \nu}{12(-\beta)^{3 / 2}|\xi|^{3 / 2} \sqrt{\frac{3}{4} \xi^{3}+\frac{1}{\beta} \nu}|\sigma+\nu+k-\mu|^{1 / 2}\langle\mu\rangle^{2 b}}\right\}^{1 / 2} \\
\leqslant & \frac{C|\xi|^{\frac{1}{4}+s}\langle\theta\rangle}{\langle\sigma\rangle^{b^{\prime}}}\left\{\int_{-3|\sigma|}^{3|\sigma|} \frac{|\nu|^{1 / 2} d \nu}{\sqrt{\frac{3}{4} \xi^{3}+\frac{1}{\beta} \nu}\langle\sigma+\nu+k\rangle^{1 / 2}}\right\}^{1 / 2} .
\end{aligned}
$$

Notice that we have $|\nu+k| \geqslant|\nu|$ and from (3.2) we know $|\sigma| \geqslant \frac{1}{3}|\nu+k| \geqslant \frac{1}{3}|\nu|$.

- If $\langle\sigma\rangle^{b_{1}} \leqslant|\xi|^{b_{2}}$, since $\min \left\{\left|\xi_{1}\right|,\left|\xi-\xi_{1}\right|\right\} \leqslant \frac{1}{10}|\xi|$, we know that $|\nu| \leqslant \frac{-3 \beta}{8}|\xi|^{3}$.

Hence $\left|-\frac{1}{\beta} \nu\right|^{1 / 2} \leqslant\left|\frac{3}{4} \xi^{3}+\frac{1}{\beta} \nu\right|^{1 / 2} \sim|\xi|^{3 / 2}$. Therefore

$$
\begin{aligned}
I(\tau, \zeta) & \leqslant \frac{C|\xi|^{\frac{1}{4}+s}}{\langle\sigma\rangle^{\frac{1}{4}}}\left\{\int_{-3|\sigma|}^{3|\sigma|} \frac{|\nu|^{1 / 2} d \nu}{|\nu|^{2 b^{\prime}-\frac{1}{2}} \sqrt{\frac{3}{4} \xi^{3}+\frac{1}{\beta} \nu}\langle\sigma+\nu+k\rangle^{1 / 2}}\right\}^{1 / 2} \\
& \leqslant \frac{C|\xi|^{\frac{1}{4}+s}}{\langle\sigma\rangle^{\frac{1}{4}}}\left\{\int_{-3|\sigma|}^{3|\sigma|} \frac{|\xi|^{3-6 b^{\prime}} d \nu}{|\xi|^{3 / 2}\langle\sigma+\nu+k\rangle^{1 / 2}}\right\}^{1 / 2} \\
& \leqslant \frac{C|\xi|^{\frac{1}{4}+s}}{\langle\xi\rangle^{3 b^{\prime}-\frac{3}{4}}} \leqslant C, \quad \text { since } b^{\prime}>\frac{1+s}{3}
\end{aligned}
$$

where we have used the fact that

Claim 3.1.

$$
\int_{-3|\sigma|}^{3|\sigma|} \frac{d \nu}{\langle\sigma+\nu+k\rangle^{1 / 2}} \leqslant C|\sigma|^{1 / 2}, \quad \text { where } C=C(\gamma) .
$$

Recall that $k=\frac{A}{\nu}$ is defined in (3.4).

Proof of (3.6). Let $f(\nu)=\nu+\frac{A}{\nu}$, where $A$ is defined in (3.5), and let $\nu_{1} \leqslant \nu_{2}$ be such that

$$
f\left(\nu_{1}\right)=f\left(\nu_{2}\right)=\sigma, \text { for } \sigma \geqslant 2 \sqrt{A} .
$$

Without loss of generality we may assume that $\sigma \geqslant 0$. Then we know that for $\nu>0$,

$$
\sigma+\nu+k \geqslant \nu>0
$$

and hence

$$
\int_{0}^{3 \sigma} \frac{d \nu}{\langle\sigma+\nu+k\rangle^{1 / 2}} \leqslant \int_{0}^{3 \sigma} \frac{d \nu}{\nu^{1 / 2}} \leqslant C \sqrt{\sigma}
$$

Therefore we only need to consider the term $\int_{-3 \sigma}^{0} \frac{d \nu}{\langle\sigma+\nu+k\rangle^{1 / 2}}$. By a change of variable $\nu \rightarrow-\nu$, it is equivalent to consider the integral

$$
\int_{0}^{3 \sigma} \frac{d \nu}{\langle\sigma-\nu-k\rangle^{1 / 2}}
$$

From the definition of $A$ and (3.2) we know that

$$
A \leqslant C \gamma \sigma \text {. }
$$


(1) If $\sigma \leqslant 2 \sqrt{A}$, then from (3.9) we know that $\sigma \leqslant C \gamma$, and hence

$$
\int_{0}^{3 \sigma} \frac{d \nu}{\langle\sigma-\nu-k\rangle^{1 / 2}} \leqslant 3 \sigma \leqslant C \sqrt{\sigma}
$$

(2) If $\sigma>2 \sqrt{A}$, then we know that $f\left(\nu_{1}\right)=f\left(\nu_{2}\right)=\sigma$ and $0<\nu_{1}<$ $\sqrt{A}<\nu_{2}$. Moreover, we have that $f$ is decreasing on $(0, \sqrt{A}]$ and increasing on $[\sqrt{A}, \sigma)$. Also on $(0, \sqrt{A}]$,

$$
\int_{0}^{2 \sqrt{A}} \frac{d \nu}{\langle\sigma-\nu-k\rangle^{1 / 2}} \leqslant 2 \sqrt{A} \leqslant C \sqrt{\sigma} .
$$

If $\nu_{2}<2 \sqrt{A}$, then

$$
\sigma=f\left(\nu_{2}\right)<f(2 \sqrt{A})=\frac{5}{2} \sqrt{A} \leqslant C \sqrt{\sigma} .
$$

Then using the same argument as before we have

$$
\int_{0}^{3 \sigma} \frac{d \nu}{\langle\sigma-\nu-k\rangle^{1 / 2}} \leqslant 3 \sigma \leqslant C \sqrt{\sigma} .
$$

If $\nu_{2} \geqslant 2 \sqrt{A}$, then on $\left[2 \sqrt{A}, \nu_{2}\right]$,

$$
\frac{f(\nu)-f\left(\nu_{2}\right)}{\nu-\nu_{2}} \geqslant f^{\prime}(\nu) \geqslant f^{\prime}(2 \sqrt{A})=\frac{3}{4} .
$$

Thus on this interval

$$
\sigma-f(\nu) \geqslant \frac{3}{4}\left(\nu_{2}-\nu\right)
$$

and then

$$
\int_{2 \sqrt{A}}^{\nu_{2}} \frac{d \nu}{\langle\sigma-\nu-k\rangle^{1 / 2}} \leqslant C \int_{2 \sqrt{A}}^{\nu_{2}} \frac{d \nu}{\left(\nu_{2}-\nu\right)^{1 / 2}} \leqslant C \sqrt{\sigma} .
$$

Similarly, on $\left[\nu_{2}, 3 \sigma\right]$,

$$
\frac{f(\nu)-f\left(\nu_{2}\right)}{\nu-\nu_{2}} \geqslant f^{\prime}\left(\nu_{2}\right) \geqslant f^{\prime}(2 \sqrt{A})=\frac{3}{4} .
$$

Therefore we also have

$$
\int_{\nu_{2}}^{3 \sigma} \frac{d \nu}{\langle\sigma-\nu-k\rangle^{1 / 2}} \leqslant C \sqrt{\sigma}
$$

Summing over the above argument, we obtain

$$
\int_{0}^{3 \sigma} \frac{d \nu}{\langle\sigma-\nu-k\rangle^{1 / 2}} \leqslant C \sqrt{\sigma}
$$

Combining (3.8) and (3.10) we have proved (3.6).

- If $\langle\sigma\rangle^{b_{1}} \geqslant|\xi|^{b_{2}}$, then similarly we get

$$
\begin{aligned}
I(\tau, \zeta) & \leqslant \frac{C|\xi|^{\frac{1}{4}+s-b_{2}}}{\langle\sigma\rangle^{\frac{1}{4}}}\left\{\int_{-3|\sigma|}^{3|\sigma|} \frac{|\nu|^{1-2 b^{\prime}+2 b_{1}} d \nu}{\sqrt{\frac{3}{4} \xi^{3}+\frac{1}{\beta} \nu}\langle\sigma+\nu+k\rangle^{1 / 2}}\right\}^{1 / 2} \\
& \leqslant \frac{C|\xi|^{\frac{1}{4}+s-b_{2}}}{\langle\xi\rangle^{3\left(b^{\prime}-b_{1}-\frac{1}{4}\right)}} \leqslant C,
\end{aligned}
$$

since $b^{\prime}-b_{1}>\frac{1}{4}$ and $b^{\prime}-b_{1}>\frac{1+s-b_{2}}{3}$. 
In this way we know that $I(\tau, \zeta)$ is bounded for large $|\xi|$. Using the Cauchy-Schwarz inequality to (3.3), we get

$$
J_{212} \leqslant C\|u\|_{L^{2}}\|v\|_{L^{2}}\|w\|_{L^{2}} .
$$

Case 2.2: $|\sigma| \geqslant\left|\sigma_{1}\right|$ and $\min \left\{\left|\xi_{1}\right|,\left|\xi-\xi_{1}\right|\right\} \leqslant 1$. In this case we have

$$
\langle\xi\rangle^{-s}\left\langle\xi_{1}\right\rangle^{s}\left\langle\xi-\xi_{1}\right\rangle^{s} \leqslant C
$$

and we can use the same argument as in Case 2.1.2 with $s=0$.

Case 2.3: $|\sigma| \leqslant\left|\sigma_{1}\right|$ and $\left|\xi_{1}\right| \geqslant 1$.

Case 2.3.1: $|\xi| \leqslant 2\left|\xi_{1}\right|$. In this case we have

$$
\frac{\langle\theta\rangle}{\left\langle\theta_{1}\right\rangle} \leqslant C \frac{\left\langle\xi_{1}\right\rangle^{b_{2}}}{\langle\xi\rangle^{b_{2}}} \text { and }\langle\xi\rangle^{-s}\left\langle\xi_{1}\right\rangle^{s}\left\langle\xi-\xi_{1}\right\rangle^{s} \leqslant C \frac{\left\langle\xi_{1}\right\rangle^{2 s}}{\langle\xi\rangle^{s}} .
$$

Thus

$$
K\left(\tau, \zeta, \tau_{1}, \zeta_{1}\right) \leqslant \frac{C|\xi|\left\langle\xi_{1}\right\rangle^{b_{2}+2 s}}{\langle\sigma\rangle^{b^{\prime}}\left\langle\sigma_{1}\right\rangle^{b}\left\langle\sigma_{2}\right\rangle^{b}} \frac{1}{\langle\xi\rangle^{b_{2}+s}} .
$$

Since $|\sigma| \leqslant\left|\sigma_{1}\right|$ and $b^{\prime}<b$, we know

$$
\langle\sigma\rangle^{b^{\prime}}\left\langle\sigma_{1}\right\rangle^{b} \geqslant\langle\sigma\rangle^{b}\left\langle\sigma_{1}\right\rangle^{b^{\prime}}
$$

and

$$
\frac{\left\langle\xi_{1}\right\rangle^{b_{2}+2 s}}{\langle\xi\rangle^{b_{2}+s-1}} \leqslant C\left\langle\xi_{1}\right\rangle^{1+s}
$$

for $b_{2}+s \leqslant 1$. Thus

$$
K\left(\tau, \zeta, \tau_{1}, \zeta_{1}\right) \leqslant \frac{C\left|\xi_{1}\right|^{1+s}}{\left\langle\sigma_{1}\right\rangle^{b^{\prime}}\langle\sigma\rangle^{b}\left\langle\sigma_{2}\right\rangle^{b}} .
$$

By replacing $(\tau, \zeta)$ with $\left(\tau_{1}, \zeta_{1}\right)$, we can use the same argument as in Case 2.1.2 to obtain the right bound.

Case 2.3.2: $|\xi| \geqslant 2\left|\xi_{1}\right|$. Then $|\xi| \leqslant 2\left|\xi-\xi_{1}\right|$ and $\langle\xi\rangle^{-s}\left\langle\xi_{1}\right\rangle^{s}\left\langle\xi-\xi_{1}\right\rangle^{s} \leqslant C\left\langle\xi_{1}\right\rangle^{s}$. Hence

$$
K\left(\tau, \zeta, \tau_{1}, \zeta_{1}\right) \leqslant \frac{C|\xi|\left|\xi_{1}\right|^{s}\langle\theta\rangle}{\langle\sigma\rangle^{b^{\prime}}\left\langle\sigma_{1}\right\rangle^{b}\left\langle\sigma_{2}\right\rangle^{b}\left\langle\theta_{1}\right\rangle} .
$$

- $|\sigma|^{b_{1}} \leqslant|\xi|^{b_{2}}$. Since $\left|\sigma_{1}\right|$ dominates in (3.2), we obtain

$$
K\left(\tau, \zeta, \tau_{1}, \zeta_{1}\right) \leqslant \frac{C|\xi|\left|\xi_{1}\right|^{b_{2}+s}}{\langle\sigma\rangle^{b^{\prime}}\left\langle\sigma_{1}\right\rangle^{b+b_{1}}\left\langle\sigma_{2}\right\rangle^{b}} \leqslant \frac{C}{\langle\sigma\rangle^{b^{\prime}}\left\langle\sigma_{2}\right\rangle^{b}\left\langle\sigma_{1}\right\rangle^{b+b_{1}-\max \left\{\frac{1}{2}, \frac{1+s+b_{2}}{3}\right\}}} .
$$

Since $b>\frac{1}{2}$ and $b^{\prime}+b_{1}-\max \left\{\frac{1}{2}, \frac{1+s+b_{2}}{3}\right\}>0$, we know that $b^{\prime}+b+b+$ $b_{1}-\max \left\{\frac{1}{2}, \frac{1+s+b_{2}}{3}\right\}>1$. Therefore we can use Lemma 2.8 to obtain the right bound.

- $|\sigma|^{b_{1}} \geqslant|\xi|^{b_{2}}$. Similarly,

$$
K\left(\tau, \zeta, \tau_{1}, \zeta_{1}\right) \leqslant \frac{C|\xi|^{1-b_{2}}\left|\xi_{1}\right|^{b_{2}+s}}{\langle\sigma\rangle^{b^{\prime}-b_{1}}\left\langle\sigma_{1}\right\rangle^{b+b_{1}}\left\langle\sigma_{2}\right\rangle^{b}} \leqslant \frac{C}{\langle\sigma\rangle^{b^{\prime}-b_{1}}\left\langle\sigma_{2}\right\rangle^{b}\left\langle\sigma_{1}\right\rangle^{b+b_{1}-\max \left\{\frac{1-b_{2}}{2}, \frac{1+s}{3}\right\}}} .
$$

Since $b>\frac{1}{2}$ and $b^{\prime}-\max \left\{\frac{1-b_{2}}{2}, \frac{1+s}{3}\right\}>0$, we know that $b^{\prime}-b_{1}+b+b+b_{1}-$ $\max \left\{\frac{1-b_{2}}{2}, \frac{1+s}{3}\right\}>1$. Therefore we can use Lemma 2.8 to give the right bound. 
Case 2.4: $|\sigma| \leqslant\left|\sigma_{1}\right|$ and $\left|\xi_{1}\right| \leqslant 1$. Denote by $J_{24}$ the contribution of this region to $J$. On the support of $J_{24}$ we know that

$$
\langle\xi\rangle^{-s}\left\langle\xi_{1}\right\rangle^{s}\left\langle\xi-\xi_{1}\right\rangle^{s} \leqslant C
$$

Hence we can assume that $s=0$. Now consider the dyadic levels

$$
\mathcal{A}^{K M}=\left\{\left(\tau_{1}, \zeta_{1}\right):\left|\xi_{1}\right| \sim M,\left\langle\sigma_{1}\right\rangle \sim K\right\},
$$

where $K=2^{k}, k=0,1,2, \ldots$, and $M=2^{m}, m=0,-1,-2, \ldots$ Denote by $J_{24}^{K M}$ the contribution of $\mathcal{A}^{K M}$ to $J_{24}$; then

$$
J_{24} \leqslant C \sum_{K, M} J_{24}^{K M}
$$

Using Cauchy-Schwarz we have

$$
J_{24}^{K M} \leqslant C \int I^{K M}(\tau, \zeta)\left\{\int\left|\hat{u}\left(\tau_{1}, \zeta_{1}\right) \hat{v}\left(\tau-\tau_{1}, \zeta-\zeta_{1}\right)\right|^{2} d \tau_{1} d \zeta_{1}\right\}^{1 / 2} \hat{w}(\tau, \zeta) d \tau d \zeta,
$$

where

$$
I^{K M}(\tau, \zeta)=\frac{|\xi|\langle\theta\rangle}{\langle\sigma\rangle^{b^{\prime}}}\left(\int_{\mathcal{A}^{K M}} \frac{d \tau_{1} d \zeta_{1}}{\left\langle\sigma_{1}\right\rangle^{2 b}\left\langle\sigma_{2}\right\rangle^{2 b}\left\langle\theta_{1}\right\rangle^{2}\left\langle\theta_{2}\right\rangle^{2}}\right)^{1 / 2} .
$$

Similar to Case 2.1.2, we perform the change of variables

$$
\nu=-3 \beta \xi \xi_{1}\left(\xi-\xi_{1}\right), \quad \mu=\sigma_{1}+\sigma_{2} .
$$

We can assume that $\xi \geqslant 0$ and then

$$
|\nu| \leqslant-3 \beta|\xi|(|\xi|+1) \cdot 2 M \leqslant-9 \beta|\xi|^{2} M .
$$

Since $b^{\prime} \geqslant b_{1}$, we have $\langle\theta\rangle \leqslant C\langle\sigma\rangle^{b^{\prime}}$. Hence

$$
\begin{aligned}
& I^{K M}(\tau, \zeta) \leqslant\left(\int_{-\infty}^{\infty} \int_{-\infty}^{\infty} \int_{9 \beta|\xi|^{2} M}^{-9 \beta|\xi|^{2} M}\right. \\
& \left.\quad \times \frac{C|\xi|^{1 / 2}|\nu|^{1 / 2} d \nu d \tau_{1} d \mu}{12(-\beta)^{3 / 2}\left\langle\sigma_{1}\right\rangle^{2 b}\left\langle\mu-\sigma_{1}\right\rangle^{2 b} \sqrt{\frac{3}{4} \xi^{3}+\frac{1}{\beta} \nu}|(\sigma+\nu+k)-\mu|^{1 / 2}\left\langle\theta_{1}\right\rangle^{2}}\right)^{1 / 2} .
\end{aligned}
$$

From (3.2) we know $|\nu| \leqslant 3\left|\sigma_{1}\right|$, and therefore

$$
\frac{|\nu|^{1 / 2}}{\left\langle\theta_{1}\right\rangle^{2}} \leqslant \frac{C\left\langle\sigma_{1}\right\rangle^{1 / 2}\left\langle\xi_{1}\right\rangle^{2 b_{2}}}{\left\langle\sigma_{1}\right\rangle^{2 b_{1}}} \leqslant C\left\langle\sigma_{1}\right\rangle^{\frac{1}{2}-2 b_{1}} \leqslant C K^{\frac{1}{2}-2 b_{1}} .
$$

In this way, using (2.20) and (2.21),

$$
\begin{aligned}
I^{K M}(\tau, \zeta) & \leqslant C|\xi|^{\frac{1}{4}} K^{\frac{1}{4}-b_{1}}\left\{\int_{-\infty}^{\infty} \int_{9 \beta|\xi|^{2} M}^{-9 \beta|\xi|^{2} M} \frac{d \nu d \mu}{\langle\mu\rangle^{2 b}\left(\frac{3}{4} \xi^{3}+\frac{1}{\beta} \nu\right)^{1 / 2}|(\sigma+\nu+k)-\mu|^{1 / 2}}\right\}^{\frac{1}{2}} \\
& \leqslant C|\xi|^{\frac{1}{4}} K^{\frac{1}{4}-b_{1}}\left\{\int_{9 \beta|\xi|^{2} M}^{-9 \beta|\xi|^{2} M} \frac{d \nu}{\left(\frac{3}{4} \xi^{3}+\frac{1}{\beta} \nu\right)^{1 / 2}\langle\sigma+\nu+k\rangle^{1 / 2}}\right\}^{\frac{1}{2}} .
\end{aligned}
$$

Since $|\xi|$ is large and $\left|\xi_{1}\right|$ is small, we have from (3.2) that $|\nu| \leqslant \frac{-3 \beta}{8}|\xi|^{3}$, and hence $\sqrt{\frac{3}{4} \xi^{3}+\frac{1}{\beta} \nu} \geqslant \frac{3}{8}|\xi|^{3}$. Therefore

$$
I^{K M}(\tau, \zeta) \leqslant \frac{C K^{\frac{1}{4}-b_{1}}}{|\xi|^{\frac{1}{2}}}\left\{\int_{9 \beta|\xi|^{2} M}^{-9 \beta|\xi|^{2} M} \frac{d \nu}{\langle\sigma+\nu+k\rangle^{1 / 2}}\right\}^{\frac{1}{2}}
$$


Claim 3.2.

$$
\int_{9 \beta|\xi|^{2} M}^{-9 \beta|\xi|^{2} M} \frac{d \nu}{\langle\sigma+\nu+k\rangle^{1 / 2}} \leqslant C M^{\frac{1}{4}}|\xi| .
$$

Proof of (3.12). Let $a=-9 \beta|\xi|^{2} M$. Without loss of generality we may assume $a \geqslant 1$. Thus $a \geqslant \sqrt{a}$. Similarly as in the proof of (3.6), we can assume $\sigma>0$. Hence

$$
\int_{0}^{a} \frac{d \nu}{\langle\sigma+\nu+k\rangle^{1 / 2}} \leqslant \int_{0}^{a} \frac{d \nu}{\nu^{1 / 2}} \leqslant \sqrt{a} \leqslant C M^{\frac{1}{2}}|\xi| \leqslant C M^{\frac{1}{4}}|\xi|
$$

since $M \leqslant 1$. Therefore we only need to show

$$
\int_{0}^{a} \frac{d \nu}{\langle\sigma-\nu-k\rangle^{1 / 2}} \leqslant C M^{\frac{1}{4}}|\xi|
$$

Since now $|\xi| \geqslant 8$ and $\left|\xi_{1}\right| \leqslant 1$, we have

$$
-3 \beta \gamma \xi^{2} \leqslant A \leqslant-\frac{21}{8} \beta \gamma \xi^{2},
$$

where $A$ is defined in (3.5). Therefore we obtain that

$$
\frac{3}{\gamma} M A \leqslant a \leqslant \frac{24}{7 \gamma} M A
$$

Denote

$$
c_{1}=\sqrt{\frac{24}{7 \gamma}}, \quad c_{2}=\sqrt{\frac{3}{\gamma}} .
$$

(1) Consider the case $a \geqslant A$. When $a \leqslant 2 \sqrt{A}$, then

$$
\int_{0}^{a} \frac{d \nu}{\langle\sigma-\nu-k\rangle^{1 / 2}} \leqslant 2 \sqrt{A} \leqslant 2 \sqrt{a} \leqslant C M^{\frac{1}{2}}|\xi| \leqslant C M^{\frac{1}{4}}|\xi| .
$$

Thus we only check when $a>2 \sqrt{A}$.

(1-1) If $\sigma<2 \sqrt{A}$, we have on $[2 \sqrt{A}, a]$ that

$$
f(\nu)-\sigma \geqslant f(\nu)-f(2 \sqrt{A}) \geqslant f^{\prime}(2 \sqrt{A})(\nu-2 \sqrt{A})=\frac{3}{4}(\nu-2 \sqrt{A}) .
$$

Thus

$$
\int_{2 \sqrt{A}}^{a} \frac{d \nu}{\langle\sigma-\nu-k\rangle^{1 / 2}} \leqslant C \sqrt{a} \leqslant C M^{\frac{1}{4}}|\xi| .
$$

(1-2) If $\sigma \geqslant 2 \sqrt{A}$, then

$$
\int_{0}^{2 \sqrt{A}} \frac{d \nu}{\langle\sigma-\nu-k\rangle^{1 / 2}} \leqslant 2 \sqrt{A} \leqslant C M^{\frac{1}{4}}|\xi| .
$$

When $\nu_{2}<2 \sqrt{A}$, then on $[2 \sqrt{A}, a]$, where $\nu_{2}$ is defined in (3.7),

$$
f(\nu)-\sigma=f(\nu)-f\left(\nu_{2}\right) \geqslant f(\nu)-f(2 \sqrt{A}) \geqslant f^{\prime}(2 \sqrt{A})(\nu-2 \sqrt{A})=\frac{3}{4}(\nu-2 \sqrt{A}),
$$

and then

$$
\int_{2 \sqrt{A}}^{a} \frac{d \nu}{\langle\sigma-\nu-k\rangle^{1 / 2}} \leqslant C \sqrt{a} \leqslant C M^{\frac{1}{4}}|\xi| .
$$

When $\nu_{2} \geqslant 2 \sqrt{A}$, then $f^{\prime}\left(\nu_{2}\right) \geqslant f^{\prime}(2 \sqrt{A})=\frac{3}{4}$. On $\left[2 \sqrt{A}, \nu_{2}\right]$,

$$
\sigma-f(\nu) \geqslant f^{\prime}(\nu)\left(\nu_{2}-\nu\right) \geqslant f^{\prime}(2 \sqrt{A})\left(\nu_{2}-\nu\right) .
$$


On $\left[\nu_{2}, a\right]$ we have

$$
f(\nu)-\sigma \geqslant f^{\prime}\left(\nu_{2}\right)\left(\nu-\nu_{2}\right) \geqslant f^{\prime}(2 \sqrt{A})\left(\nu-\nu_{2}\right) .
$$

So

$$
\int_{2 \sqrt{A}}^{a} \frac{d \nu}{\langle\sigma-\nu-k\rangle^{1 / 2}} \leqslant C \sqrt{a} \leqslant C M^{\frac{1}{4}}|\xi| .
$$

(2) Now we consider the case $a<A$. In this case we have from (3.15) that

$$
M<\frac{\gamma}{3}, \text { and thus } \sqrt{M}<\frac{1}{c_{2}} .
$$

Thus we have the following estimates for the derivatives:

$$
\begin{aligned}
-f^{\prime}(\sqrt{A}-\sqrt{a}) & =\frac{A}{(\sqrt{A}-\sqrt{a})^{2}}-1 \geqslant \frac{1}{\left(1-c_{1} \sqrt{M}\right)^{2}}-1 \\
& \geqslant \frac{1}{1-c_{1} \sqrt{M}}-1 \geqslant c_{1} \sqrt{M}, \\
f^{\prime}(\sqrt{A}+\sqrt{a}) & =1-\frac{A}{(\sqrt{A}+\sqrt{a})^{2}} \geqslant 1-\frac{1}{\left(1+c_{2} \sqrt{M}\right)^{2}} \\
& \geqslant 1-\frac{1}{1+c_{2} \sqrt{M}}=\frac{c_{2} \sqrt{M}}{1+c_{2} \sqrt{M}} \geqslant \frac{c_{2}}{2} \sqrt{M},
\end{aligned}
$$

where we have used the estimate (3.17).

(2-1) If $\sigma<2 \sqrt{A}$, we know that on $[0, \sqrt{A}-\sqrt{a}]$,

$$
\begin{aligned}
f(\nu)-\sigma & \geqslant f(\nu)-f(\sqrt{A}-\sqrt{a}) \geqslant-f^{\prime}(\sqrt{A}-\sqrt{a})[(\sqrt{A}-\sqrt{a})-\nu] \\
& \geqslant c_{1} \sqrt{M}[(\sqrt{A}-\sqrt{a})-\nu] .
\end{aligned}
$$

So we have

$$
\int_{0}^{\sqrt{A}-\sqrt{a}} \frac{d \nu}{\langle\sigma-\nu-k\rangle^{1 / 2}} \leqslant C M^{-\frac{1}{4}}(\sqrt{A}-\sqrt{a})^{\frac{1}{2}} .
$$

Hence if $a \leqslant \sqrt{A}-\sqrt{a}$, then

$$
\int_{0}^{a} \frac{d \nu}{\langle\sigma-\nu-k\rangle^{1 / 2}} \leqslant C M^{-\frac{1}{4}} \sqrt{a}=C M^{\frac{1}{4}}|\xi| .
$$

When $a>\sqrt{A}-\sqrt{a}$, we have

$$
\begin{aligned}
\int_{0}^{a} \frac{d \nu}{\langle\sigma-\nu-k\rangle^{1 / 2}} & =\left(\int_{0}^{\sqrt{A}-\sqrt{a}}+\int_{\sqrt{A}-\sqrt{a}}^{a}\right) \frac{d \nu}{\langle\sigma-\nu-k\rangle^{1 / 2}} \\
& \leqslant C M^{-\frac{1}{4}} \sqrt{a}+\sqrt{a} \leqslant C M^{\frac{1}{4}}|\xi| .
\end{aligned}
$$

In either case we obtain (3.12).

When $a>\sqrt{A}$, we have on $[\sqrt{A}+\sqrt{a}, a]$,

$$
\begin{aligned}
f(\nu)-\sigma & \geqslant f(\nu)-f(\sqrt{A}+\sqrt{a}) \geqslant-f^{\prime}(\sqrt{A}+\sqrt{a})[\nu-(\sqrt{A}+\sqrt{a})] \\
& \geqslant \frac{c_{2}}{2} \sqrt{M}[\nu-(\sqrt{A}+\sqrt{a})],
\end{aligned}
$$

and thus

$$
\int_{\sqrt{A}+\sqrt{a}}^{a} \frac{d \nu}{\langle\sigma-\nu-k\rangle^{1 / 2}} \leqslant C M^{-\frac{1}{4}} \sqrt{a} .
$$


Therefore combining (3.20) and (3.21) we get

$$
\begin{aligned}
\int_{0}^{a} \frac{d \nu}{\langle\sigma-\nu-k\rangle^{1 / 2}} & =\left(\int_{0}^{\sqrt{A}-\sqrt{a}}+\int_{\sqrt{A}-\sqrt{a}}^{\sqrt{A}+\sqrt{a}}+\int_{\sqrt{A}+\sqrt{a}}^{a}\right) \frac{d \nu}{\langle\sigma-\nu-k\rangle^{1 / 2}} \\
& \leqslant C M^{-\frac{1}{4}}(\sqrt{A}-\sqrt{a})^{\frac{1}{2}}+2 \sqrt{a}+C M^{-\frac{1}{4}} \sqrt{a} \\
& \leqslant C M^{-\frac{1}{4}} \sqrt{a} \leqslant C M^{\frac{1}{4}}|\xi|,
\end{aligned}
$$

which is (3.14).

(2-2) When $\sigma \geqslant 2 \sqrt{A}$, we discuss the following cases.

$(2-2-1) a \leqslant \sqrt{A}$.

(2-2-1-1) If $a \leqslant \sqrt{A}-\sqrt{a}$, then when $\nu_{1} \leqslant a$ we have that

$$
\begin{aligned}
f(\nu)-\sigma & =f(\nu)-f\left(\nu_{1}\right) \geqslant-f^{\prime}\left(\nu_{1}\right)\left(\nu_{1}-\nu\right) \geqslant-f^{\prime}(\sqrt{A}-\sqrt{a})\left(\nu_{1}-\nu\right) \\
& \geqslant c_{1} \sqrt{M}\left(\nu_{1}-\nu\right), \quad \nu \in\left[0, \nu_{1}\right], \\
\sigma-f(\nu) & =f\left(\nu_{1}\right)-f(\nu) \geqslant-f^{\prime}\left(\nu_{1}\right)\left(\nu-\nu_{1}\right) \geqslant-f^{\prime}(\sqrt{A}-\sqrt{a})\left(\nu-\nu_{1}\right) \\
& \geqslant c_{1} \sqrt{M}\left(\nu-\nu_{1}\right), \quad \nu \in\left[\nu_{1}, a\right] .
\end{aligned}
$$

Hence we know

$$
\int_{0}^{a} \frac{d \nu}{\langle\sigma-\nu-k\rangle^{1 / 2}}=\left(\int_{0}^{\nu_{1}}+\int_{\nu_{1}}^{a}\right) \frac{d \nu}{\langle\sigma-\nu-k\rangle^{1 / 2}} \leqslant C M^{-\frac{1}{4}} \sqrt{a} \leqslant C M^{\frac{1}{4}}|\xi| .
$$

When $\nu_{1}>a$, we have

$$
\begin{aligned}
f(\nu)-\sigma & \geqslant f(\nu)-f(a) \geqslant-f^{\prime}(a)(a-\nu) \\
& \geqslant-f^{\prime}(\sqrt{A}-\sqrt{a})(a-\nu) \geqslant c_{1} \sqrt{M}(a-\nu),
\end{aligned}
$$

and then

$$
\int_{0}^{a} \frac{d \nu}{\langle\sigma-\nu-k\rangle^{1 / 2}} \leqslant C M^{-\frac{1}{4}} \sqrt{a} \leqslant C M^{\frac{1}{4}}|\xi| .
$$

(2-2-1-2) If $\sqrt{A}-\sqrt{a}<a \leqslant \sqrt{A}$, then when $\nu_{1}<\sqrt{A}-\sqrt{a}$ we have (3.22) on $\left[0, \nu_{1}\right]$ and $(3.23)$ on $\left[\nu_{1}, \sqrt{A}-\sqrt{a}\right]$. Thus

$$
\begin{aligned}
\int_{0}^{a} \frac{d \nu}{\langle\sigma-\nu-k\rangle^{1 / 2}} & =\left(\int_{0}^{\nu_{1}}+\int_{\nu_{1}}^{\sqrt{A}-\sqrt{a}}+\int_{\sqrt{A}-\sqrt{a}}^{a}\right) \frac{d \nu}{\langle\sigma-\nu-k\rangle^{1 / 2}} \\
& \leqslant C M^{-\frac{1}{4}} \sqrt{a}+\sqrt{a} \leqslant C M^{\frac{1}{4}}|\xi| .
\end{aligned}
$$

If $\sqrt{A}-\sqrt{a} \leqslant \nu_{1}$, then on $[0, \sqrt{A}-\sqrt{a}]$,

$$
\begin{aligned}
f(\nu)-\sigma & \geqslant f(\nu)-f(\sqrt{A}-\sqrt{a}) \geqslant-f^{\prime}(\sqrt{A}-\sqrt{a})[(\sqrt{A}-\sqrt{a})] \\
& \geqslant c_{1} \sqrt{M}[(\sqrt{A}-\sqrt{a})] .
\end{aligned}
$$

Therefore

$$
\begin{aligned}
\int_{0}^{a} \frac{d \nu}{\langle\sigma-\nu-k\rangle^{1 / 2}} & =\left(\int_{0}^{\sqrt{A}-\sqrt{a}}+\int_{\sqrt{A}-\sqrt{a}}^{a}\right) \frac{d \nu}{\langle\sigma-\nu-k\rangle^{1 / 2}} \\
& \leqslant C M^{-\frac{1}{4}} \sqrt{a}+\sqrt{a} \leqslant C M^{\frac{1}{4}}|\xi| .
\end{aligned}
$$


$(2-2-2)$ If $a>\sqrt{A}$, we first look at

$$
\int_{0}^{\sqrt{A}} \frac{d \nu}{\langle\sigma-\nu-k\rangle^{1 / 2}}
$$

In this situation we consider the two cases $\nu_{1}<\sqrt{A}-\sqrt{a}$ or $\nu_{1} \geqslant \sqrt{A}-\sqrt{a}$. We may use the same argument as in (2-2-1-2) to obtain the desired estimate (3.14).

Now for

$$
\int_{\sqrt{A}}^{a} \frac{d \nu}{\langle\sigma-\nu-k\rangle^{1 / 2}}
$$

when $\nu_{2}<\sqrt{A}+\sqrt{a}$, we have on $[\sqrt{A}+\sqrt{a}, a]$ that

$$
\begin{aligned}
f(\nu)-\sigma & \geqslant f(\nu)-f(\sqrt{A}+\sqrt{a}) \geqslant f^{\prime}(\sqrt{A}+\sqrt{a})[\nu-(\sqrt{A}+\sqrt{a})] \\
& \geqslant \frac{c_{2}}{2} \sqrt{M}[\nu-(\sqrt{A}+\sqrt{a})] .
\end{aligned}
$$

Thus

$$
\begin{aligned}
\int_{\sqrt{A}}^{a} \frac{d \nu}{\langle\sigma-\nu-k\rangle^{1 / 2}} & =\left(\int_{\sqrt{A}}^{\sqrt{A}+\sqrt{a}}+\int_{\sqrt{A}+\sqrt{a}}^{a}\right) \frac{d \nu}{\langle\sigma-\nu-k\rangle^{1 / 2}} \\
& \leqslant \sqrt{a}+C M^{-\frac{1}{4}} \sqrt{a} \leqslant C M^{\frac{1}{4}}|\xi| .
\end{aligned}
$$

When $\nu_{2} \geqslant \sqrt{A}+\sqrt{a}$, in the case $\nu_{2} \leqslant a$, we know that on $\left[\sqrt{A}+\sqrt{a}, \nu_{2}\right]$,

$$
\sigma-f(\nu) \geqslant f^{\prime}(\nu)\left(\nu_{2}-\nu\right) \geqslant f^{\prime}(\sqrt{A}+\sqrt{a})\left(\nu_{2}-\nu\right) \geqslant \frac{c_{2}}{2} \sqrt{M}\left(\nu_{2}-\nu\right) .
$$

On $\left[\nu_{2}, a\right]$,

$$
f(\nu)-\sigma \geqslant f^{\prime}\left(\nu_{2}\right)\left(\nu-\nu_{2}\right) \geqslant f^{\prime}(\sqrt{A}+\sqrt{a})\left(\nu-\nu_{2}\right) \geqslant \frac{c_{2}}{2} \sqrt{M}\left(\nu-\nu_{2}\right) .
$$

So

$$
\begin{aligned}
\int_{\sqrt{A}}^{a} \frac{d \nu}{\langle\sigma-\nu-k\rangle^{1 / 2}} & =\left(\int_{\sqrt{A}}^{\sqrt{A}+\sqrt{a}}+\int_{\sqrt{A}+\sqrt{a}}^{\nu_{2}}+\int_{\nu_{2}}^{a}\right) \frac{d \nu}{\langle\sigma-\nu-k\rangle^{1 / 2}} \\
& \leqslant \sqrt{a}+C M^{-\frac{1}{4}} \sqrt{a} \leqslant C M^{\frac{1}{4}}|\xi| .
\end{aligned}
$$

When $\nu_{2}>a$, on $[\sqrt{A}+\sqrt{a}, a]$, we have

$$
\sigma-f(\nu) \geqslant f(a)-f(\nu) \geqslant f^{\prime}(\nu)(a-\nu) \geqslant f^{\prime}(\sqrt{A}+\sqrt{a})(a-\nu) \geqslant \frac{c_{2}}{2} \sqrt{M}(a-\nu) .
$$

Hence

$$
\begin{aligned}
\int_{\sqrt{A}}^{a} \frac{d \nu}{\langle\sigma-\nu-k\rangle^{1 / 2}} & =\left(\int_{\sqrt{A}}^{\sqrt{A}+\sqrt{a}}+\int_{\sqrt{A}+\sqrt{a}}^{a}\right) \frac{d \nu}{\langle\sigma-\nu-k\rangle^{1 / 2}} \\
& \leqslant \sqrt{a}+C M^{-\frac{1}{4}} \sqrt{a} \leqslant C M^{\frac{1}{4}}|\xi| .
\end{aligned}
$$

Therefore summing up all the above we obtain (3.14), and hence (3.12).

Hence using (3.12) we obtain

$$
I^{K M}(\tau, \zeta) \leqslant \frac{C K^{\frac{1}{4}-b_{1}}}{|\xi|^{\frac{1}{2}}} M^{\frac{1}{8}}|\xi|^{\frac{1}{2}} \leqslant C K^{\frac{1}{4}-b_{1}} M^{\frac{1}{8}} .
$$

Therefore in this case, by using Cauchy-Schwarz we have

$$
J_{24}^{K M} \leqslant C K^{\frac{1}{4}-b_{1}} M^{\frac{1}{8}}\|u\|_{L^{2}}\|v\|_{L^{2}}\|w\|_{L^{2}} .
$$


Here we gain a small factor $M^{\frac{1}{4}}$. To lower $b_{1}$ as much as possible in order to weaken the restriction of Case 1 , we need to apply Lemma 2.8 to estimate $J_{24}^{K M}$.

- If $|\sigma|^{b_{1}} \leqslant|\xi|^{b_{2}}$. Denote by $J_{241}^{K M}$ the contribution of this region to $J_{24}^{K M}$. Since $\left|\xi_{1}\right| \leqslant 1$, using (3.2) we get $\left|\sigma_{1}\right| \geqslant C|\xi|^{2}\left|\xi_{1}\right|$, and then

$$
|\xi| \leqslant C\left\langle\sigma_{1}\right\rangle^{1 / 2}\left|\xi_{1}\right|^{-1 / 2} \text {. }
$$

Thus

$$
K\left(\tau, \zeta, \tau_{1}, \zeta_{1}\right) \leqslant \frac{C|\xi|}{\langle\sigma\rangle^{b^{\prime}}\left\langle\sigma_{1}\right\rangle^{b+b_{1}}\left\langle\sigma_{2}\right\rangle^{b}} \leqslant \frac{C M^{-1 / 2} K^{-\delta_{1}}}{\langle\sigma\rangle^{b^{\prime}}\left\langle\sigma_{1}\right\rangle^{b+b_{1}-\frac{1}{2}-\delta_{1}}\left\langle\sigma_{2}\right\rangle^{b}},
$$

where $\delta_{1}>0$ will be specified later. Using Lemma 2.8 we obtain the bound

$$
J_{241}^{K M} \leqslant \frac{C}{M^{1 / 2} K^{\delta_{1}}}\|u\|_{L^{2}}\|v\|_{L^{2}}\|w\|_{L^{2}}
$$

provided that

$$
2 b+b^{\prime}+b_{1}-\frac{1}{2}-\delta_{1}>1 .
$$

- If $|\sigma|^{b_{1}} \geqslant|\xi|^{b_{2}}$. Denote by $J_{242}^{K M}$ the contribution of this region to $J_{24}^{K M}$. Then

$$
K\left(\tau, \zeta, \tau_{1}, \zeta_{1}\right) \leqslant \frac{C|\xi|^{1-b_{2}}}{\langle\sigma\rangle^{b^{\prime}-b_{1}}\left\langle\sigma_{1}\right\rangle^{b+b_{1}}\left\langle\sigma_{2}\right\rangle^{b}} \leqslant \frac{C M^{-\frac{1-b_{2}}{2}} K^{-\delta_{2}}}{\langle\sigma\rangle^{b^{\prime}}\left\langle\sigma_{1}\right\rangle^{b-\frac{1-b_{2}}{2}-\delta_{2}}\left\langle\sigma_{2}\right\rangle^{b}},
$$

where $\delta_{2}$ will be specified later. A use of Lemma 2.8 gives

$$
J_{242}^{K M} \leqslant \frac{C}{M^{\left(1-b_{2}\right) / 2} K^{\delta_{2}}}\|u\|_{L^{2}}\|v\|_{L^{2}}\|w\|_{L^{2}},
$$

provided that

$$
2 b+b^{\prime}-\frac{1-b_{2}}{2}-\delta_{2}>1
$$

The factors $K^{-\delta_{1}}$ and $K^{-\delta_{2}}$ in (3.25) and (3.27) help us to lower $b_{1}$.

(i) An interpolation between (3.24) and (3.25) with weights $\frac{4}{5}+$ and $\frac{1}{5}-$, respectively, yield

$$
J_{241}^{K M} \leqslant \frac{C M^{\delta_{1}^{*}}}{K^{\delta_{2}^{*}}}\|u\|_{L^{2}}\|v\|_{L^{2}}\|w\|_{L^{2}}
$$

with $\delta_{1}^{*}, \delta_{2}^{*}>0$ and

$$
\left(\frac{1}{4}-b_{1}\right)\left(\frac{4}{5}+\right)-\delta_{1}\left(\frac{1}{5}-\right)<0, \quad \text { that is, } \delta_{1}>1-4 b_{1} .
$$

(ii) An interpolation between (3.24) and (3.27) with weights $\frac{4-4 b_{2}}{5-4 b_{2}}+$ and $\frac{1}{5-4 b_{2}}-$, respectively, yield

$$
J_{242}^{K M} \leqslant \frac{C M^{\delta_{1}^{*}}}{K_{2}^{\delta_{2}^{*}}}\|u\|_{L^{2}}\|v\|_{L^{2}}\|w\|_{L^{2}}
$$

with $\delta_{1}^{*}, \delta_{2}^{*}>0$ and

$\left(\frac{1}{4}-b_{1}\right)\left(\frac{4-4 b_{2}}{5-4 b_{2}}+\right)-\delta_{2}\left(\frac{1}{5-4 b_{2}}-\right)<0$, that is, $\delta_{2}>\left(1-4 b_{1}\right)\left(1-b_{2}\right)$. 
We also know from (3.26) and (3.28) that

$$
\delta_{1}<2 b+b^{\prime}+b_{1}-\frac{3}{2}, \quad \delta_{2}<2 b+b^{\prime}+\frac{b_{2}}{2}-\frac{3}{2} .
$$

Note that since we have

$$
b>\frac{1}{2}, b^{\prime}+5 b_{1}>\frac{3}{2}, b^{\prime}+4 b_{1}+\frac{3}{2} b_{2}-4 b_{1} b_{2}>\frac{3}{2},
$$

we are able to choose the proper $\delta_{1}, \delta_{2}$ to satisfy the above conditions. Therefore, summing over $K$ and $M$ we obtain

$$
J_{24} \leqslant C\|u\|_{L^{2}}\|v\|_{L^{2}}\|w\|_{L^{2}} .
$$

This completes the proof of Theorem 1.2

\section{LOCAL WELL-POSEDNESS}

We will apply a Picard fixed-point argument to the integral equation (1.8).

Let $\psi(t)$ be a cut-off function such that $\psi \in C_{0}^{\infty}\left(\mathbb{R}^{2}\right), \operatorname{supp} \psi \subset[-2,2]$, and $\psi=1$ on $[-1,1]$. For $T>0$, let $\psi_{T}(t)=\psi(t / T)$, and define the "temporally truncated" operator

$$
L u(t)=\psi(t) S(t) \phi-\psi_{T}(t) \int_{0}^{t} S\left(t-t^{\prime}\right) \partial_{x}\left(u^{2}\left(t^{\prime}\right)\right) d t^{\prime} .
$$

Now define the space $Y^{b, s_{1}, s_{2}}$ to be the space equipped with the norm

$$
\|u\|_{Y^{b, s_{1}, s_{2}}}=\left\|\langle\tau-p(\xi, \eta)\rangle^{b}\langle\xi\rangle^{s_{1}}\langle\eta\rangle^{s_{2}} \hat{u}(\tau, \xi, \eta)\right\|_{L_{\tau \xi \eta}^{2}} .
$$

Then we can write the norm in $X_{s_{1}, s_{2}}^{b, b_{1}, b_{2}}$ as

$$
\|u\|_{X_{s_{1}, s_{2}}^{b, b_{1}, b_{2}}} \sim\|u\|_{Y^{b, s_{1}, s_{2}}}+\|u\|_{Y^{b+b_{1}, s_{1}-b_{2}, s_{2}}} .
$$

Using Lemma 2.1 and Lemma 2.3 in [9] we have

Lemma 4.1. Let $0<\epsilon<\frac{1}{4}, b=\frac{1}{2}+\epsilon, b^{\prime}=\frac{1}{2}-2 \epsilon$, and $s_{1}, s_{2} \in \mathbb{R}$. Then

$$
\begin{aligned}
& \|\psi(t) S(t) \phi\|_{Y^{b, s_{1}, s_{2}}} \leqslant C\|\phi\|_{H^{s_{1}, s_{2}}}, \\
& \left\|\psi_{T} \int_{0}^{t} S\left(t-t^{\prime}\right) f\left(u\left(t^{\prime}\right)\right) d t^{\prime}\right\|_{Y^{b, s_{1}, s_{2}}} \leqslant C T^{\epsilon}\|f(u)\|_{Y^{-b^{\prime}, s_{1}, s_{2}}} .
\end{aligned}
$$

From (4.2) and Lemma 4.1 we get

Proposition 4.2. Assume $\beta<0$ and $\gamma>0$. Let $0<\epsilon<\frac{1}{4}-\left(\frac{1}{5}+\right), b=\frac{1}{2}+\epsilon$, $b^{\prime}=\frac{1}{2}-2 \epsilon, b_{1}=\frac{1}{5}+, b_{2}>\frac{2}{7}$, and $s_{1}, s_{2} \in \mathbb{R}$. Then

$$
\|L u\|_{X_{s_{1}, s_{2}}^{b, b_{1}, b_{2}}} \leqslant C\|\phi\|_{H^{s_{1}, s_{2}}}+C T^{\epsilon}\left\|u u_{x}\right\|_{X_{s_{1}, s_{2}}^{-b^{\prime}, b_{1}, b_{2}}} .
$$

Proof. From (4.2) and Lemma 4.1 we have

$$
\begin{aligned}
\|L u\|_{X_{s_{1}, s_{2}}^{b, b_{1}, b_{2}}} \sim & \|L u\|_{Y^{b, s_{1}, s_{2}}}+\|L u\|_{Y^{b+b_{1}, s_{1}-b_{2}, s_{2}}} \\
\leqslant & C\left(\|\phi\|_{H^{s_{1}, s_{2}}}+\|\phi\|_{H^{s_{1}-b_{2}, s_{2}}}\right) \\
& +C T^{\epsilon}\left(\left\|u u_{x}\right\|_{Y^{-b^{\prime}, s_{1}, s_{2}}}+\left\|u u_{x}\right\|_{Y^{-b^{\prime}+b_{1}, s_{1}-b_{2}, s_{2}}}\right) \\
\leqslant & C\|\phi\|_{H^{s_{1}, s_{2}}}+C T^{\epsilon}\left\|u u_{x}\right\|_{X_{s_{1}, s_{2}}^{-b^{\prime}, b_{1}, b_{2}}} .
\end{aligned}
$$

Hence the proposition is proved.

Now we can prove Theorem 1.4 on the local well-posedness. 
Proof of Theorem 1.4. Using Theorem 1.3 with $b=\frac{1}{2}+\epsilon, b^{\prime}=\frac{1}{2}-2 \epsilon$, and Proposition 4.2 we obtain

$$
\begin{aligned}
& \|L u\|_{X_{s_{1}, s_{2}}^{1 / 2+, 1 / 6+, 1 / 3}} \leqslant C\|\phi\|_{H^{s_{1}, s_{2}}}+C T^{\epsilon}\|u\|_{X_{s_{1}, s_{2}}^{1 / 2+, 1 / 6+, 1 / 3}}^{2}, \\
& \|L u-L v\|_{X_{s_{1}, s_{2}}^{1 / 2+, 1 / 6+, 1 / 3}} \leqslant C T^{\epsilon}\|u+v\|_{X_{s_{1}, s_{2}}^{1 / 2,1 / 6+, 1 / 3}}\|u-v\|_{X_{s_{1}, s_{2}}^{1 / 2+, 1 / 6+, 1 / 3}} .
\end{aligned}
$$

Choosing

$$
T=\frac{1}{\left(5 C^{2}\|\phi\|_{H^{s_{1}, s_{2}}}\right)^{1 / \epsilon}},
$$

we deduce from (4.4) and (4.5) that the mapping $L$ is strictly contractive on the ball of radius $2 C\|\phi\|_{H^{s_{1}, s_{2}}}$ in $X_{s_{1}, s_{2}}^{1 / 2+1 / 6+, 1 / 3}$. This gives the existence and uniqueness of a solution to the truncated problem (4.1), and hence also proves the existence of the solution $u \in X_{s_{1}, s_{2}}^{1 / 2+, 1 / 6+, 1 / 3}$ to the full integral equation (1.8) on the time interval $[-T, T]$ with $T=T\left(\|\phi\|_{H^{s_{1}, s_{2}}}\right)$. Furthermore, choosing $T$ small enough to make $\psi, \psi_{T}=1$ on $I=[-T, T]$, we conclude the local existence and uniqueness of the solution to (1.8).

To prove the continuity of the flow map $\phi \mapsto u$, we consider $u_{1}, u_{2}$ to be two solutions on $[-T, T]$ with initial data $\phi_{1}$ and $\phi_{2}$, respectively. Then

$$
\begin{aligned}
\left\|u_{1}-u_{2}\right\|_{X_{s_{1}, s_{2}}^{1 / 2+, 1 / 6+, 1 / 3}} \leqslant & C\left\|\phi_{1}-\phi_{2}\right\|_{H^{s_{1}, s_{2}}} \\
& +C T^{\epsilon}\left\|u_{1}+u_{2}\right\|_{X_{s_{1}, s_{2}}^{1 / 2+1 / 6+, 1 / 3}}\left\|u_{1}-u_{2}\right\|_{X_{s_{1}, s_{2}}^{1 / 2+, 1 / 6+, 1 / 3}},
\end{aligned}
$$

which immediately implies the Lipschitz continuity of the flow map.

\section{Exponential DECAY OF SOLITARY WAVES}

This section is concerned with the exponential decay of the solitary waves to equation (1.1).

Let $H^{\infty}\left(\mathbb{R}^{2}\right)=\bigcap_{m \in \mathbb{N}} H^{m}\left(\mathbb{R}^{2}\right)$. Inspired by the argument in [6], we have the following regularity results for solitary waves.

Proposition 5.1. Assume $\beta, \gamma>0$ and $c<2 \sqrt{\beta \gamma}$. Then any solution $\varphi$ of (1.16) belongs to $C^{\infty}\left(\mathbb{R}^{2}\right) \cap H^{\infty}\left(\mathbb{R}^{2}\right)$. Moreover, $D_{x}^{-1} \varphi$ and all its derivatives decay to zero at infinity.

We will first set up an integral decay estimate for the solitary waves. Let $B_{r}$ denote the ball in $\mathbb{R}^{2}$ with radius $r$ centered in origin and let

$$
\Omega_{n}=\mathbb{R}^{2} \backslash B_{n L}
$$

be domains for positive integers $n$ and $L>1$, a number to be determined later. We have

Lemma 5.2. Under the assumption of Theorem 1.6, for any solitary wave $\varphi$ of (1.1) there exist positive numbers $C=C(\beta, c, \gamma, \varphi)$ and $\alpha=\alpha(\beta, c, \gamma)$ such that

$$
\int_{\Omega_{n}} \varphi_{x x}^{2}+\varphi_{x}^{2}+\varphi_{y}^{2}+\varphi^{2} \leqslant C e^{-2 \alpha n L}
$$

is valid for every positive integer $n$.

Proof. Let $L>0$ as stated in (5.1). For each positive integer $n$ we set $\chi_{n}(r)$ : $(0,+\infty) \rightarrow[0,1]$ to be a piecewise smooth affine function such that

$$
\chi_{n}(r) \equiv 0 \quad \text { if } r \leqslant n L, \quad \chi_{n}(r) \equiv 1 \quad \text { if } r \geqslant(n+1) L
$$


and for $n L<r<(n+1) L$

$$
\left|\chi_{n}^{\prime}(r)\right|,\left|\chi_{n}^{\prime \prime}(r)\right| \leqslant \frac{4}{L}
$$

Replacing $r$ by $\sqrt{x^{2}+y^{2}}$, we consider $\chi_{n}$ as a function of $(x, y)$ in plane $\mathbb{R}^{2}$. For simplicity of notation let $\chi_{n, x x}, \chi_{n, x}$ and $\chi_{n, y}$ be the second partial derivative of $\chi_{n}$ with respect to $x$ and the first derivatives to $x$ and $y$, respectively. By the choice of $\chi_{n}$ we see that these partial derivatives vanish in the ball $B_{n L}$ and the exterior domain $\Omega_{n+1}$, and

$$
\left|\chi_{n, x x}(x, y)\right|,\left|\chi_{n, x}(x, y)\right|,\left|\chi_{n, y}(x, y)\right| \leqslant \frac{4}{L}
$$

in annular domain $n L \leqslant r=\sqrt{x^{2}+y^{2}} \leqslant(n+1) L$.

Taking the derivative with respect to $x,(1.16)$ is rewritten as

$$
\beta \varphi_{x x x x}+c \varphi_{x x}-\varphi_{y y}+\gamma \varphi=\left(\varphi^{2}\right)_{x x} .
$$

We multiply terms of (5.4) by $\chi_{n} \varphi$ and integrate on $\mathbb{R}^{2}$, respectively. Using several integrations by parts and recalling $\chi_{n}=0$ in $B_{n L}$, it follows that

$$
\begin{aligned}
\int_{\Omega_{n}} \chi_{n} \varphi \varphi_{x x x x} & =\int_{\Omega_{n}}\left(\chi_{n} \varphi\right)_{x x} \varphi_{x x} \\
& =\int_{\Omega_{n}}\left(\chi_{n} \varphi_{x x}+2 \chi_{n, x} \varphi_{x}+\chi_{n, x x} \varphi\right) \varphi_{x x} \\
& =\int_{\Omega_{n}}\left(\chi_{n} \varphi_{x x}+\chi_{n, x x} \varphi\right) \varphi_{x x}-\chi_{n, x x} \varphi_{x}^{2}, \\
-\int_{\Omega_{n}} \chi_{n} \varphi \varphi_{x x} & =\int_{\Omega_{n}}\left(\chi_{n} \varphi\right)_{x} \varphi_{x}=\int_{\Omega_{n}} \chi_{n} \varphi_{x}^{2}-\frac{1}{2} \chi_{n, x x} \varphi^{2}, \\
-\int_{\Omega_{n}} \chi_{n} \varphi \varphi_{y y} & =\int_{\Omega_{n}}\left(\chi_{n} \varphi\right)_{y} \varphi_{y}=\int_{\Omega_{n}} \chi_{n} \varphi_{y}^{2}-\frac{1}{2} \chi_{n, y y} \varphi^{2},
\end{aligned}
$$

and

$$
\int_{\Omega_{n}} \chi_{n} \varphi\left(\varphi^{2}\right)_{x x}=\int_{\Omega_{n}}\left(\chi_{n} \varphi\right)_{x x} \varphi^{2} .
$$

Multiplying equation (5.4) by $\chi_{n} \varphi$ and integrating on $\mathbb{R}^{2}$, then using (5.5) and (5.7), we find that

$$
\begin{aligned}
\int_{\Omega_{n}} \chi_{n} & \left(\beta \varphi_{x x}^{2}+c \varphi_{x x} \varphi+\varphi_{y}^{2}+\gamma \varphi^{2}\right) \\
& =\int_{\Omega_{n}} \chi_{n} \varphi\left(\varphi^{2}\right)_{x x}+\beta \chi_{n, x x} \varphi_{x}^{2}-\beta \chi_{n, x x} \varphi \varphi_{x x}+\frac{1}{2} \chi_{n, y y} \varphi^{2} .
\end{aligned}
$$

By Proposition 5.1, it follows that $\varphi_{x x}(x, y), \varphi_{x}(x, y), \varphi(x, y) \rightarrow 0$ as $r \rightarrow+\infty$. Then $0 \leqslant \chi_{n} \leqslant 1$ and (5.3) implies that there exists a positive integer $n_{0}$ such that for $r \geqslant n_{0} L$

$$
\left|\left(\chi_{n} \varphi\right)_{x x}(x, y)\right|=\left|\left(\chi_{n} \varphi_{x x}+2 \chi_{n, x} \varphi_{x}+\chi_{n, x x} \varphi\right)(x, y)\right| \leqslant \frac{1}{L},
$$

from which along with (5.8) we have for $n \geqslant n_{0}$

$$
\left|\int_{\Omega_{n}} \chi_{n} \varphi\left(\varphi^{2}\right)_{x x}\right| \leqslant \int_{\Omega_{n}}\left|\left(\chi_{n} \varphi\right)_{x x} \varphi^{2}\right| \leqslant \frac{1}{L} \int_{\Omega_{n}} \varphi^{2} .
$$


We rewrite (5.6) and apply Hölder inequality to get

$$
\begin{aligned}
\int_{\Omega_{n}} \chi_{n} \varphi_{x}^{2} & =\int_{\Omega_{n}}-\chi_{n} \varphi \varphi_{x x}+\frac{1}{2} \chi_{n, x x} \varphi^{2} \\
& \leqslant \int_{\Omega_{n}} \frac{1}{2} \chi_{n} \varphi_{x x}^{2}+\frac{1}{2}\left(\chi_{n}+\chi_{n, x x}\right) \varphi^{2} .
\end{aligned}
$$

Now we prove (5.2) in two cases according to the sign of $c$.

(a) $0 \leqslant c<2 \sqrt{\beta \gamma}$. In this situation we see, by the Hölder inequality, that there is some positive constant $\delta$ depending only $\beta, \gamma$ and $c$ such that for all $(x, y) \in \mathbb{R}^{2}$

$$
\delta\left(\varphi_{x x}^{2}+\varphi^{2}\right) \leqslant \beta \varphi_{x x}^{2}+c \varphi_{x x} \varphi+\gamma \varphi^{2} .
$$

For simplicity of notation, let

$$
\begin{aligned}
& E_{n}=\int_{\Omega_{n}} \chi_{n}\left(\beta \varphi_{x x}^{2}+c \varphi_{x x} \varphi+\gamma \varphi^{2}+\delta \varphi_{x}^{2}+\varphi_{y}^{2}\right), \\
& F_{n}=\int_{\Omega_{n}} \beta \varphi_{x x}^{2}+c \varphi_{x x} \varphi+\gamma \varphi^{2}+\delta \varphi_{x}^{2}+\varphi_{y}^{2},
\end{aligned}
$$

where $\delta$ is determined in (5.12). Note that comparing with the left-hand side of (5.9), we add a term $\delta \varphi_{x}^{2}$ in $E_{n}$ and $F_{n}$, respectively. Recalling $\chi_{n}(x, y)=1$ in $\Omega_{n+1}$, the nonnegativity of the right-hand side of (5.12) leads to

$$
F_{n+1} \leqslant E_{n} .
$$

Applying the Hölder inequality to the third integral of the right-hand side of (5.9) and using (5.10) and (5.11), we see that $E_{n}$ is less than

$$
\begin{aligned}
\int_{\Omega_{n}} & \frac{1}{2}\left(\delta \chi_{n}+\beta\left|\chi_{n, x x}\right|\right) \varphi_{x x}^{2}+\beta\left|\chi_{n, x x}\right| \varphi_{x}^{2} \\
& +\frac{1}{2}\left(\delta \chi_{n}+(\delta+\beta)\left|\chi_{n, x x}\right|+\left|\chi_{n, y y}\right|+\frac{2}{L}\right) \varphi^{2}
\end{aligned}
$$

under the condition $n \geqslant n_{0}$. By (5.3) we can take $L$ sufficiently large such that the preceding integral, and therefore $E_{n}$, is less than

$$
\frac{3}{4} \int_{\Omega_{n}} \delta\left(\varphi_{x x}^{2}+\varphi^{2}+\varphi_{x}^{2}\right)
$$

Taking into account (5.12), we deduce for $n \geqslant n_{0}$

$$
E_{n} \leqslant \frac{3}{4} F_{n} .
$$

It is deduced from (5.13) and (5.14) that $F_{n+1} \leqslant \frac{3}{4} F_{n}$ for $n \geqslant n_{0}$, which implies for $n \geqslant n_{0}$

$$
F_{n} \leqslant\left(\frac{3}{4}\right)^{n-n_{0}} F_{n_{0}}=C e^{-2 \alpha n L}
$$

with $C=\left(\frac{3}{4}\right)^{-n_{0}} F_{n_{0}}$ and $\alpha=\frac{1}{2 L}\left|\ln \frac{3}{4}\right|$. It then follows the preceding inequality for $F_{n}$, the definition of $F_{n}$ and (5.12) that

$$
\int_{\Omega_{n}} \delta \varphi_{x x}^{2}+\delta \varphi_{x}^{2}+\delta \varphi^{2}+\varphi_{y}^{2} \leqslant C e^{-2 \alpha n L}
$$


for $n \geqslant n_{0}$. The estimate is also valid for positive integers $n<n_{0}$ if $C$ is taken to be sufficiently large. Replacing $C$ by $(\min \{1, \delta\})^{-1} C$ we have proved (5.2) in the case $0 \leqslant c<2 \sqrt{\beta \gamma}$.

(b) $c<0$. In this setting, we introduce notation

$$
\begin{aligned}
G_{n} & =\int_{\Omega_{n}} \chi_{n}\left(\beta \varphi_{x x}^{2}-c \varphi_{x}^{2}+\varphi_{y}^{2}+\gamma \varphi^{2}\right), \\
H_{n} & =\int_{\Omega_{n}} \beta \varphi_{x x}^{2}-c \varphi_{x}^{2}+\varphi_{y}^{2}+\gamma \varphi^{2} .
\end{aligned}
$$

Then $\chi_{n}(x, y)=1$ in $\Omega_{n+1}$ and $c<0$ imply

$$
H_{n+1} \leqslant G_{n} .
$$

By (5.6) and (5.9) we have

$$
\begin{aligned}
G_{n}= & \int_{\Omega_{n}} \chi_{n}\left(\beta \varphi_{x x}^{2}+c \varphi \varphi_{x x}+\varphi_{y}^{2}+\gamma \varphi^{2}\right)-\frac{c}{2} \chi_{n, x x} \varphi^{2} \\
= & \int_{\Omega_{n}} \chi_{n} \varphi\left(\varphi^{2}\right)_{x x}+\beta \chi_{n, x x} \varphi_{x}^{2}-\beta \chi_{n, x x} \varphi \varphi_{x x} \\
& +\frac{1}{2} \chi_{n, y y} \varphi^{2}-\frac{c}{2} \chi_{n, x x} \varphi^{2} .
\end{aligned}
$$

Applying Hölder inequality to the third integral of (5.16) and then using (5.10), we see that $G_{n}$ is less than

$$
\int_{\Omega_{n}} \frac{1}{2} \beta\left|\chi_{n, x x}\right| \varphi_{x x}^{2}+\beta\left|\chi_{n, x x}\right| \varphi_{x}^{2}+\frac{1}{2}\left(-c\left|\chi_{n, x x}\right|+\beta\left|\chi_{n, x x}\right|+\left|\chi_{n, y y}\right|+\frac{2}{L}\right) \varphi^{2}
$$

when $n \geqslant n_{0}$. By (5.3) we can take $L$ sufficiently large such that the preceding integral is less than $\frac{3}{4} H_{n}$. So for $n \geqslant n_{0}$,

$$
G_{n} \leqslant \frac{3}{4} H_{n}
$$

By (5.15) and (5.17) we get $H_{n+1} \leqslant \frac{3}{4} H_{n}$, from which we infer for $n \geqslant n_{0}$ that

$$
H_{n} \leqslant\left(\frac{3}{4}\right)^{n-n_{0}} H_{n_{0}}=C e^{-2 \alpha n L},
$$

with $C=\left(\frac{3}{4}\right)^{-n_{0}} H_{n_{0}}$ and $\alpha=\frac{1}{2 L}\left|\ln \frac{3}{4}\right|$ as in the first case. Then we get

$$
\int_{\Omega_{n}} \beta \varphi_{x x}^{2}-c \varphi_{x}^{2}+\varphi_{y}^{2}+\gamma \varphi^{2} \leqslant C e^{-2 \alpha n L}
$$

for $n \geqslant n_{0}$. The inequality is also valid for positive integers $n<n_{0}$ if $C$ is taken sufficiently large. Note that $c<0$ in this setting. With some modification on constant $C$, we have proved (5.2) in the case $c<0$.

Remark 5.1. Let $\mathbf{l}=\left(l_{1}, l_{2}\right)=(2,1)$ and $\mathbf{p}=\left(p_{1}, p_{2}\right)=(2,2)$. In terms of the notation and terminologies of anisotropic spaces (page 165 of [5]), we get from (5.2) that for each natural number $n$,

$$
\begin{aligned}
\|\varphi\|_{W_{\mathbf{p}}^{1}\left(\Omega_{n}\right)} & =\|\varphi\|_{\mathbf{p}, \Omega_{n}}+\left\|\varphi_{x x}\right\|_{\mathbf{p}, \Omega_{n}}+\left\|\varphi_{y}\right\|_{\mathbf{p}, \Omega_{n}} \\
& =\|\varphi\|_{L^{2}\left(\Omega_{n}\right)}+\left\|\varphi_{x x}\right\|_{L^{2}\left(\Omega_{n}\right)}+\left\|\varphi_{y}\right\|_{L^{2}\left(\Omega_{n}\right)} \\
& \leqslant C e^{-\alpha n L} .
\end{aligned}
$$


Set $\alpha=(0,0), \mathbf{1}=(1,1)$ and $\mathbf{q}=\left(q_{1}, q_{2}\right)=(\infty, \infty)$. We also see that $\mathbf{1} \leqslant \mathbf{p} \leqslant \mathbf{q}$ and that the basic index (see page 180 of [5] for a definition)

$$
\kappa=\left|\left(\alpha+\frac{1}{\mathbf{p}}-\frac{1}{\mathbf{q}}\right): \mathbf{l}\right|=\frac{1}{p_{1}} \cdot \frac{1}{l_{1}}+\frac{1}{p_{2}} \cdot \frac{1}{l_{2}}=\frac{1}{2} \cdot \frac{1}{2}+\frac{1}{2} \cdot \frac{1}{1}=\frac{3}{4}<1 .
$$

Recalling that $\varphi$ is smooth by Proposition [5.1, one has

$$
\begin{aligned}
\|\varphi\|_{L_{\mathbf{q}}\left(\Omega_{n}\right)} & =\|\tilde{\varphi}\|_{L_{\mathbf{q}}\left(\mathbb{R}_{n}\right)}=\operatorname{ess} \sup _{y \in \mathbb{R}}(\underset{x \in \mathbb{R}}{\operatorname{ess} \sup |\tilde{\varphi}(x, y)|)} \\
& =\operatorname{ess}_{(x, y) \in \mathbb{R}^{2}} \sup _{\tilde{\varphi}}(x, y) \mid=\|\varphi\|_{L^{\infty}\left(\Omega_{n}\right)},
\end{aligned}
$$

where $\tilde{\varphi}(x, y)=\varphi(x, y)$ for $(x, y) \in \Omega_{n}$ and $\tilde{\varphi}(x, y)=0$ for $(x, y) \in \mathbb{R}^{2} \backslash \Omega_{n}$.

Proof of Theorem 1.6. Note that for all positive integers $n$, the domains $\Omega_{n}$ satisfy a weak l-horm condition (page 153 of [5]) with the same parameters independent of $n$. By the generalized Sobolev imbedding theorem (see, e.g., Theorem 10.2 of [5], page 187, in the case $\alpha=(0,0)$ and $\kappa<1)$, for all positive integers $n$, the norm of $L_{\mathbf{q}}\left(\Omega_{n}\right)$ is controlled by that of $W_{\mathbf{p}}^{\mathbf{l}}\left(\Omega_{n}\right)$ with some common positive constant $C_{1}$, that is,

$$
\|\varphi\|_{L_{\mathbf{q}}\left(\Omega_{n}\right)} \leqslant C_{1}\|\varphi\|_{W_{\mathbf{p}}^{\mathbf{1}}\left(\Omega_{n}\right)} .
$$

For any $(x, y) \in \mathbb{R}^{2}$ satisfying $r=\sqrt{x^{2}+y^{2}}>L$, let $n$ be the integer such that $n L<r \leqslant(n+1) L$. This implies $(x, y) \in \Omega_{n}$. Using (5.19) and (5.20) and then (5.18) we get

$$
\begin{aligned}
|\varphi(x, y)| & \leqslant\|\varphi\|_{L^{\infty}\left(\Omega_{n}\right)} \leqslant C_{1}\|\varphi\|_{W_{\mathbf{p}}^{\mathbf{1}}\left(\Omega_{n}\right)} \\
& \leqslant C_{1} C e^{-\alpha n L}=C_{1} C e^{\alpha L} e^{-\alpha(n+1) L} \leqslant C_{1} C e^{\alpha L} e^{-\alpha r} .
\end{aligned}
$$

Theorem 1.6 is thereby established.

\section{ACKNOWLEDGments}

The authors would like to thank the referees for valuable comments and suggestions, especially in setting up estimates (3.6) and (3.12). The authors also thank Professor Vladimír Šverák and Professor Guilong Gui for many fruitful discussions. The first author was supported in part by NSF grant DMS-0908663. The second author was supported in part by NSF grant DMS-0906099 and NHARP grant 003599-0001-2009.

\section{REFERENCES}

[1] M.J. Ablowitz and H. Segur, Solitons and the inverse scattering transform, 4 SIAM Studies in Applied Mathematrics, SIAM, Philadelphia, PA, 1981. MR642018 (84a:35251)

[2] T.R. Akylas, On the radiation damping of a solitary wave in a rotating channel, Mathematical Approaches in Hydrodynamics, SIAM, Philadelphia, PA, 1991, 175-181. MR.1136562 (92h:76015)

[3] A. Ambrosetti, V. Felli, and A. Malchiodi, Ground states of nonlinear Schrödinger equations with potentials vanishing at infinity, J. Eur. Math. Soc., 7 (2005), 117-144. MR2120993 (2006f:35049)

[4] M. Ben-Artzi and J.C. Saut, Uniform decay estimates for a class of oscillatory integrals and applications, Differential Integral Equations, 12 (1999), no. 2, 137-145. MR.1672730 (2000a:35207)

[5] O.V. Besov, V.P. Il'in and S.M. Nikolskii, Integral representations of functions and imbedding theorems. I, J. Wiley, New York, 1978. MR0519341 (80f:46030a) 
[6] A. de Bouard and J.C. Saut, Solitary waves of generalized Kadomtsev-Petviashvili equations, Ann. Inst. H. Poincaré Anal. Non Linéarire, 14 (1997), 211-236. MR1441393 (98a:35115)

[7] A. de Bourad and J.C. Saut, Symmetries and decay of the generalized Kadomtsev-Petviashvili solitary waves, SIAM J. Math. Anal., 28 (1997) no. 5, 1064-1085. MR.1466669 (99c:35208)

[8] J. Bourgain, On the Cauchy problem for the Kadomtsev-Petviashvili equation, GAGA, 3 (1993), 315-341. MR1223434 (94d:35142)

[9] R.M. Chen, The Cauchy problem and the stability of solitary waves of a hyperelastic dispersive equation, Indiana U. J. Math., 57 (2008), 2377-2422. MR2463973

[10] R.M. Chen, V. Hur and Y. Liu, Solitary waves of the rotation-modified KadomtsevPetviashvili equation, Nonlinearity, 21 (2008), 2949-2979. MR2461048 (2010a:35213)

[11] A.S. Fokas and L.Y. Sung, On the solvability of the N-wave, Davey-Stewartson and Kadomtsev-Petviashvili equaitons, Inverse Probelms, 8 (1992), 673-708. MR1185594 (93h:35177)

[12] V. N. Galkin and Yu. A. Stepanyants, On the existence of stationary solitary waves in a rotating fluid (Russian), Prikl. Mat. Mekh., 55 (1991), 1051-1055; translation in J. Appl. Math. Mech., 55 (1991), 939-943. MR.1150448 (92j:76096)

[13] J. Ginibre, Le problème de Cauchy pour de EDP semi-linéaires périodiques en variables d'espace (d'après Bourgain), Séminaire Bourbaki 796, Astérisque, 237 (1995), 163-187. MR.1423623 (98e:35154)

[14] R. Grimshaw, Evolution equations for weakly nonlinear, long internal waves in a rotating fluid, Stud. Appl. Math., 73 (1985), 1-33. MR797556 (87a:76029)

[15] R.H.J. Grimshaw, L.A. Ostrovsky, V.I. Shrira and Yu.A. Stepanyants, Long nonlinear surface and internal gravity waves in a rotating ocean, Surveys in Geophysics, 19 (1998), 289-338.

[16] R. Grimshaw and S. Tang, The rotation-modified Kadomtsev-Petviashvili equation: An analytical and numerical study, Stud. Appl. Math., 83 (1990), 223-248. MR.1071361|(92a:35139)

[17] M. Hadac, S. Herr and H. Koch, Well-posedness and scattering for the KP-II equation in a critical space, Ann. Inst. H. Poincaré Anal. Non Linéaire, 26 (2009), no. 3, 917-941. MR2526409 (2010d:35301)

[18] A.D. Ionescu, C.E. Kenig and D. Tataru, Global well-posedness of the KP-I initial-value problem in the energy space, Invent. Math., 173 (2008), 265-304. MR2415308|(2009h:35368)

[19] R.J. Iório, Jr. and W.V.L. Nunes, On equations of KP-type, Proc. Roy. Soc. Edinburgh Sect. A, 128 (1998), 725-743. MR.1635416 (99j:35189)

[20] P. Isaza and J. Mejia, Local and global Cauchy problems for the Kadomtsev-Petviashvili (KPII) equation in Sobolev spaces of negative indices, Comm. Partial Differential Equations, 26 (2001), 1027-1057. MR1843294 (2002e:35205)

[21] B.B. Kadomtsev and V.I. Petviashvili, On the stability of solitary waves in weakly dispersing media, Sov. Phys. Dokl., 15 (1970), 539-541.

[22] C.E. Kenig, On the local and global well-posedness theory for the KP-I equation, Ann. Inst. Henri Poincaré, Anal. Non Linéaire, 21 (2004), 827-838. MR2097033 (2005j:35196)

[23] C.E. Kenig, G. Ponce and L. Vega, Oscillatory intergrals and regularity of dispersive equations, Indiana Univ. Math. J., 40 (1991), 33-69. MR1101221 (92d:35081)

[24] L. Molinet, J.C. Saut, and N. Tzvetkov, Well-posedness and ill-posedness results for the Kadomtsev-Petviashvili-I equation, Duke Math J., 115 (2002), 353-384. MR1944575 (2003h:35240)

[25] L. Molinet, J.C. Saut, and N. Tzvetkov, Global well-posedness for the KP-I equation, Math. Ann., 324 (2002), 255-275. MR1933858 (2003h:35241)

[26] L. Molinet, J.C. Saut, and N. Tzvetkov, Correction: Global well-posedness for the KP-I equation, Math. Ann., 328 (2004), 707-710. MR2047648 (2005b:35245)

[27] L. Molinet, J.C. Saut, and N. Tzvetkov, Remarks on the mass constraint for KP-type equations, SIAM J. Math. Anal., 39 (2007), 627-641. MR2338424 (2008h:35327)

[28] L.A. Ostrovsky, Nonlinear internal waves in a rotating ocean, Okeanologia, 18 (1978), 181191.

[29] L.A. Ostrovsky and Y.A. Stepanyants, Nonlinear waves in a rotating fluid (Russian), Nonlinear waves (Russian), 132-153, "Nauka", Moscow, 1993. MR1318473 (95k:76115)

[30] L.V. Ovsyannikov, N.I. Makarenko, V.I. Nalimov, V. Yu Lyapidevskiui, P.I. Plotnikov, I.V. Sturova, V.A. Vladimirov, Nonlinear problems in the theory of surface and internal waves (Russian), Chapter 8, "Nauka" Sibirsk. Otdel., Novosibirsk, (1985). MR851183 (87j:76002) 
[31] E. Stein, Harmonic Analysis: Real-Variable Methods, Orthogonality and Osillatory Integrals, Princeton Mathematical Series 42, Princeton University Press, 1993. MR.1232192 (95c:42002)

[32] E. Stein and G. Weiss, Introduction to Fourier Analysis in Euclidean Spaces, Princeton University Press, 1971. MR0304972 (46:4102)

[33] H. Takaoka, Global well-posedness for the Kadomtsev-Petviashvili-II equation, Discrete Contin. Dynam. Systems, 6 (2000), 483-499. MR.1739371 (2000m:35163)

[34] H. Takaoka and N. Tzvetkov, On the local regularity of the Kadomtsev-Petviashvili-II equation, Internat. Math. Res. Notices, (2001) no. 2, 77-114. MR1810481(2001j:35243)

[35] M.M. Tom, On a generalized Kadomtsev-Petviashvili equation, Contemporary Mathematics AMS, 200 (1996), 193-210. MR.1410508 (97e:35165)

[36] P. Tomas, A restriction theorem for the Fourier transform, Bull. A.M.S., 81 (1975), 477-478. MR0358216 (50:10681)

[37] N. Tzvetkov, Global low regularity solutions for Kadomtsev-Petviashvili equation, Differential and Integral Equations, 13 (2000), 1289-1320. MR.1787069 (2001g:35227)

[38] X. Zhou, Inverse scattering transform for the time dependent Schrödinger equation with applications to the KPI equation, Comm. Math. Phys., 128 (1990), 551-564. MR.1045884 $(91 \mathrm{j}: 34138)$

School of Mathematics, University of Minnesota, Minneapolis, Minnesota 55455

E-mail address: chenm@math.umn.edu

Current address: Department of Mathematics, University of Pittsburgh, Pittsburgh, Pennsylvania 15260

E-mail address: mingchen@pitt.edu

Department of Mathematics, University of Texas at Arlington, Arlington, Texas 76019-0408

E-mail address: yliu@uta.edu

Department of Mathematics, Jiangsu University, Jiangsu 212013, People's Republc OF CHINA

E-mail address: pzzhang@ujs.edu.cn 